\title{
MULTIDIMENSIONAL LIMIT THEOREMS FOR SMOOTHED EXTREME VALUE ESTIMATES OF POINT PROCESSES BOUNDARIES
}

\author{
Ludovic Menneteau ${ }^{1}$
}

\begin{abstract}
In this paper, we give sufficient conditions to establish central limit theorems and moderate deviation principle for a class of support estimates of empirical and Poisson point processes. The considered estimates are obtained by smoothing some bias corrected extreme values of the point process. We show how the smoothing permits to obtain Gaussian asymptotic limits and therefore pointwise confidence intervals. Some unidimensional and multidimensional examples are provided.
\end{abstract}

Mathematics Subject Classification. Primary 60G70; Secondary 62M30, 62G05.

Received March 11, 2005. Revised June 26, 2006 and March 26, 2007.

\section{INTRODUCTION}

The problem of estimating a set $S$ given a sequence of finite sets $S_{n}$ of random points drawn from its interior arises in classification [15], clustering problems [21], discriminant analysis [2], outliers detection [6], image analysis [25] and in econometrics [4]. In many cases, the unknown support can be written

$$
S=\{(x, y): x \in E ; 0 \leq y \leq f(x)\},
$$

where $f$ is an unknown function and $E$ an arbitrary set (typically a subset of $\mathbb{R}^{d}$ ). Then, the problem reduces to estimating $f$.

In econometrics, the data consist of pairs $\left(X_{i}, Y_{i}\right)$ where $X_{i}$ represents the input, possibly multidimensional (labor, energy or capital), used to produce an output $Y_{i}$ in a given firm. In such a framework, the value $f(x)$ can be interpreted as the maximum level of output which is attainable for the level of input $x$. Then, economical considerations suggest to suppose that $f$ is increasing and concave and an adapted estimator, called the DEA (Data Envelopment Analysis) has been developed and studied. Its asymptotic distribution is established by [10].

In the case where we do not assume that $f$ is monotone, Geffroy [9] proposed an estimator of $f$ which is a kind of histogram based on the extreme values of the sample. Geffroy's estimator has been improved in several directions. On one hand, piecewise polynomials estimators were introduced and their optimality in an asymptotic minimax sense was proved under different regularity conditions on $f$ (see $[17,20,25-27,33]$ ).

On the other hand, regularization and bias correction of Geffroy's estimate have been considered using different way of smoothing (see $[8,11,12]$ ). In [13], the multivariate central limit theorem has been obtained for a general class of estimates of this type including all the above. For technical reason (the maxima on disjoint

Keywords and phrases. Functional estimate, central limit theorem, moderate deviation principles, extreme values, shape estimation.

1 Place Eugène Bataillon, 34095 Montpellier Cedex 5, France; mennet@math.univ-montp2.fr 
cell of Poisson processes are independent) the results obtained in $[8,11,12]$ and [13] were only given when $S_{n}$ is generated by a Poisson point process. This paper encompasses the results obtained in [13] of several manners. Central limit theorems but also moderate deviation principles are investigated for a wider class of estimators than in [13]. Moreover, we show that our results hold in the context of Poisson process but also when $S_{n}$ is generated by an empirical point process.

In the next section, the general class of estimators is given. Section 2 contains our main results and provides some examples of possible bias reduction. Some applications, including kernel and projection estimators are provided in Section 3. The proofs are given in Section 4.

\section{The Boundary EStimate}

Let $f:(E, \mathcal{E}) \rightarrow\left(\mathbb{R}^{+}, \mathcal{B}\left(\mathbb{R}^{+}\right)\right)$be a measurable function on a probability space $(E, \mathcal{E}, \nu)$, where $\mathcal{B}\left(\mathbb{R}^{+}\right)$is the Borel $\sigma$-algebra on $\mathbb{R}^{+}$. Consider the set

$$
S=\{(x, y) \in E \times \mathbb{R}, 0 \leq y \leq f(x)\} .
$$

Our aim is to estimate $S$ from a sequence of $S$-valued random vectors

$$
S_{n}=\left\{\left(X_{n, i}, Y_{n, i}\right), 1 \leq i \leq N_{n}(S)\right\},
$$

with associated counting process

$$
N_{n}=\left\{N_{n}(D): D \in \mathcal{E} \otimes \mathcal{B}\left(\mathbb{R}^{+}\right)\right\}, n \geq 1,
$$

of mean measure

$$
n c \mathbf{1}_{S}(x, y) \nu(\mathrm{d} x) \mathrm{d} y,
$$

where $c>0$. Two cases are considered below:

(P) $N_{n}$ is a Poisson point process,

(E) $N_{n}$ is an ( $n$-sample) empirical point process.

Let $k_{n} \uparrow \infty$ and denote by $\left\{I_{n, r}: 1 \leq r \leq k_{n}\right\}$ a measurable partition of $E$. For all $1 \leq r \leq k_{n}$, set

$$
\nu_{n, r}=\nu\left(I_{n, r}\right), \quad D_{n, r}=\left\{(x, y): x \in I_{n, r}, 0 \leq y \leq f(x)\right\} \quad \text { and } \quad N_{n, r}=N_{n}\left(D_{n, r}\right) .
$$

We also introduce

$$
Y_{n, r}^{*}=\max \left\{Y_{n, i}:\left(X_{n, i}, Y_{n, i}\right) \in D_{n, r}\right\},
$$

if $D_{n, r} \neq \emptyset$ and $Y_{n, r}^{*}=0$ otherwise (in the sequel, we use the convention $0 \times \infty=0$ ) and

$$
f_{n, r}=\nu_{n, r}^{-1} \int_{I_{n, r}} f \mathrm{~d} \nu
$$

denotes the mean value of $f$ on $I_{n, r}$.

In that context, $f$ can be estimated using Geffroy estimator

$$
\bar{f}_{n}(x)=\sum_{r=1}^{k_{n}} \mathbf{1}_{I_{n, r}}(x) Y_{n, r}^{*}
$$

But several improvements of $\bar{f}_{n}$ can be considered. First, it is well-known that $Y_{n, r}^{*}$ estimates $f_{n, r}$ with a negative bias which reveals to be of the order $\left(n c \nu_{n, r}\right)^{-1}$. Hence, to reduce this bias we can introduce unbiased 
versions of ( 3$)$ :

$$
\widehat{f}_{n}^{\circ}\left(x ; \widehat{c}_{n}\right)=\sum_{r=1}^{k_{n}} \mathbf{1}_{I_{n, r}}(x)\left(Y_{n, r}^{*}+\left(n \widehat{c}_{n} \nu_{n, r}\right)^{-1}\right)
$$

where $\widehat{c}_{n}$ is a convenient estimator of $c$. Now, the non random conterpoint of $\widehat{f}_{n}^{\circ}$ is the step function

$$
f_{n}^{\circ}(x)=\sum_{r=1}^{k_{n}} \mathbf{1}_{I_{n, r}}(x) f_{n, r}
$$

and if $f$ is regular, it is clear that smoothed version of (5) can lead to more efficient estimation of $f$.

Therefore, we will consider estimators of $f$ of the form

$$
\widehat{f}_{n}\left(x ; \widehat{c}_{n}\right)=\sum_{r=1}^{k_{n}} \nu_{n, r} \kappa_{n, r}(x)\left(Y_{n, r}^{*}+\left(n \widehat{c}_{n}(x) \nu_{n, r}\right)^{-1}\right) \quad(x \in E),
$$

where $\kappa_{n, r}: E \rightarrow \mathbb{R}$ is a weighting function determining the nature of the smoothing introduced in the estimate. In the next section, some general conditions are imposed on $\kappa_{n, r}$ and $\widehat{c}_{n}$ in order to obtain a central limit and a moderate deviation principle for $\widehat{f}_{n}$.

\section{MAin RESUlts}

In the following, we consider the $\nu$-essential infimum and supremum of $f$ on $E$,

$$
m=\sup \{\alpha>0: \nu(\{f<\alpha\})=0\} \text { and } M=\inf \{\alpha>0: \nu(\{f>\alpha\})=0\}
$$

and, for all $1 \leq r \leq k_{n}$, we denote by

$$
\begin{aligned}
& m_{n, r}=\sup \left\{\alpha>0: \nu\left(\{f<\alpha\} \cap I_{n, r}\right)=0\right\}, \\
& M_{n, r}=\inf \left\{\alpha>0: \nu\left(\{f>\alpha\} \cap I_{n, r}\right)=0\right\}
\end{aligned}
$$

respectively the $\nu$-essential infimum, supremum of $f$ on $I_{n, r}$ (in most of applications, $E$ is a subset of $\mathbb{R}^{d}, \nu$ is absolutely continuous with respect to Lebesgue measure and $f$ is continuous, hence all ess-inf and ess-sup considered below are just classical inf and sup). For all $x \in E$, we set

$$
\begin{array}{cc}
f_{n}(x)=\sum_{r=1}^{k_{n}} \nu_{n, r} \kappa_{n, r}(x) f_{n, r}, & \kappa_{n}(x)=\left(\sum_{r=1}^{k_{n}} \kappa_{n, r}^{2}(x)\right)^{1 / 2} \\
w_{n, r}(x)=\kappa_{n, r}(x) / \kappa_{n}(x) \quad \text { and } \quad & \nu_{n}=\min \left\{\nu_{n, r}, 1 \leq r \leq k_{n}\right\} .
\end{array}
$$

In the sequel, $\left(\varepsilon_{n}\right)_{n \geq 1}$ denotes a sequence of positive real numbers such that $\left(\varepsilon_{n}\right)_{n \geq 1} \equiv 1$ or $\varepsilon_{n} \downarrow \infty$. The following assumptions will be needed below:

(H.1) $k_{n} \uparrow \infty$ and $\left(n \nu_{n}\right)^{-1} \max \left(\log (n), \varepsilon_{n}^{-1}\right) \rightarrow 0$ as $n \rightarrow \infty$.

(H.2) $0<m \leq M<+\infty$ and

$$
\delta_{n}:=\max _{1 \leq r \leq k_{n}} \nu_{n, r}\left(M_{n, r}-m_{n, r}\right)=o(1 / n) \text { as } n \rightarrow \infty
$$

There exists $F \subset E$ such that: 
(H.3) For each $\left\{x_{1}, \ldots, x_{p}\right\} \subset F$, there exists a covariance matrix $\Sigma_{\left(x_{1}, \ldots x_{p}\right)}=\left[\sigma\left(x_{i}, x_{j}\right)\right]_{1 \leq i, j \leq p}$ in $\mathbb{R}^{p}$ such that for all $1 \leq i, j \leq p$,

(H.4) For all $x \in F$,

$$
\sum_{r=1}^{k_{n}} w_{n, r}\left(x_{i}\right) w_{n, r}\left(x_{j}\right) \rightarrow \sigma\left(x_{i}, x_{j}\right) \text { as } n \rightarrow \infty
$$

(H.5) For all $x \in F$,

$$
\varepsilon_{n}^{-1 / 2} \max _{1 \leq r \leq k_{n}}\left|w_{n, r}(x)\right| \rightarrow 0 \text { as } n \rightarrow \infty .
$$

(H.6) For all $x \in F$,

$$
\varepsilon_{n}^{1 / 2} n \kappa_{n}(x)^{-1}\left|f_{n}(x)-f(x)\right| \rightarrow 0 \text { as } n \rightarrow \infty .
$$

(C.1) For all $x \in F$, and any $\eta>0$,

$$
\varepsilon_{n}^{1 / 2} \sum_{r=1}^{k_{n}}\left|w_{n, r}(x)\right|\left(n \delta_{n}\right)^{2}=o(1) \text { as } n \rightarrow \infty .
$$

$$
\limsup _{n \rightarrow \infty} \varepsilon_{n} \log \mathbb{P}\left(\varepsilon_{n}^{1 / 2}\left|\sum_{r=1}^{k_{n}} w_{n, r}(x)\right|\left|\widehat{c}_{n}(x)^{-1}-c^{-1}\right| \geq \eta\right)=-\infty .
$$

(C.2) For all $x \in F$, and any $\eta>0$,

$$
\limsup _{n \rightarrow \infty} \varepsilon_{n} \log \mathbb{P}\left(\left|\widehat{c}_{n}(x)-c\right| \geq \eta\right)=-\infty .
$$

Before proceeding, let us comment on the assumptions. (H.1)-(H.4) are devoted to the control of the conditionally centered estimator $\widehat{f}_{n}-\mathbb{E}\left(\widehat{f}_{n} \mid N_{n}\right)$. Assumption (H.1) imposes that the number of terms of the partition goes to infinity not to quikly so that the mean number of points in each $D_{n, r}$ goes to infinity. (H.2) requires the unknown function $f$ to be bounded away from 0 . It also imposes that the mean number of points in $D_{n, r}$ above $m_{n, r}$ converges to 0 . Note that (H.1) and (H.2) force the $\nu$-essential oscillation of $f$ on $I_{n, r}$ to converge uniformly to $0: \delta_{n} \rightarrow 0$ as $n \rightarrow \infty$. (H.3) is devoted to the multivariate aspects of the limit theorems. (H.4) imposes to the weight functions $\kappa_{n, r}(x)$ in the linear combination (6) to be approximatively of the same order. This is a natural condition to obtain an asymptotic gaussian behavior. These assumptions are easy to verify in practice since they involve either $f$ or $\kappa_{n, r}$ without mixing these two quantities. Assumptions (H.5) and (H.6) are devoted to the control of the residual conditional bias term $\mathbb{E}\left(\widehat{f}_{n} \mid N_{n}\right)-f$. (H.5) is natural since it implies that the non random part of the bias $f_{n}-f$ vanishes. (H.6) control of the residual conditional bias term $\mathbb{E}\left(\widehat{f}_{n} \mid N_{n}\right)-f_{n}$. These two assumptions involve both the unknown function $f$ and the weight functions $\kappa_{n, r}$ and (H.6) can be looked at as a stronger version of (H.2). Condition (C.1) imposes the speed of convergence of $\widehat{c}_{n}$ to the unknown parameter $c$ to cancel the bias terms $\left(n c \nu_{n, r}\right)^{-1}$. It appears as a minimal condition needed to estimate $c$ in the debiasing procedure without affecting the limit properties of $\widehat{f}_{n}$ in terms of central limit theorem and moderate deviation principle. Assumption (C.2) allows to replace $c$ by its estimator in the asymptotic variance of $\widehat{f}_{n}$.

\subsection{Central limit theorem}

Our first result states the multivariate central limit theorem for $\widehat{f}_{n}$ :

Theorem 2.1. Assume that (H.1) - (H.6) hold for $\left(\varepsilon_{n}\right)_{n \geq 1} \equiv 1$, that (C.1) holds for $\widehat{c}_{1, n}$ and that (C.2) holds for $\widehat{c}_{2, n}$. Then, for all $\left\{x_{1}, \ldots, x_{p}\right\} \subset F$,

$$
\left\{\kappa_{n}\left(x_{j}\right)^{-1} n \widehat{c}_{2, n}\left(x_{j}\right)\left(\widehat{f}_{n}\left(x_{j}, \widehat{c}_{1, n}\right)-f\left(x_{j}\right)\right): 1 \leq j \leq p\right\} \underset{\mathcal{D}}{\rightarrow} N\left(0, \Sigma_{\left(x_{1}, \ldots x_{p}\right)}\right),
$$


where $\underset{\mathcal{D}}{\rightarrow}$ denotes the convergence in distribution and $N\left(0, \Sigma_{\left(x_{1}, \ldots x_{p}\right)}\right)$ is the centered gaussian law in $\mathbb{R}^{p}$, with covariance matrix $\Sigma_{\left(x_{1}, \ldots x_{p}\right)}$.

This result can be used to obtain explicit asymptotic $\gamma \%$ confidence interval for $f(x)$ of the form:

$$
\left[\widehat{f}_{n}\left(x, \widehat{c}_{1, n}\right)-z_{\gamma} \kappa_{n}(x)\left(n \widehat{c}_{2, n}(x)\right)^{-1}, \widehat{f}_{n}\left(x, \widehat{c}_{1, n}\right)+z_{\gamma} \kappa_{n}(x)\left(n \widehat{c}_{2, n}(x)\right)^{-1}\right]
$$

where $z_{\gamma}$ is the $(\gamma+1) / 2$ th quantile of the $N(0,1)$ distribution.

\subsection{Moderate deviation principle}

We will now establish a family of large deviation principles for $\widehat{f}_{n}$ which is sometimes referenced in the litterature as a moderate deviation principle. Recall that a sequence of random variable $\left(W_{n}\right)_{n \geq 1}$ is said to follow the large deviation principle in $\mathbb{R}^{p}$ with speed $\varepsilon_{n} \downarrow 0$ and good rate function $\mathrm{I}: \mathbb{R}^{p} \mapsto[0, \infty]$, whenever, for every set $A \in \mathcal{B}\left(\mathbb{R}^{p}\right)$,

$$
\begin{aligned}
-\inf \{\mathrm{I}(u): u \in \stackrel{\circ}{A}\} & \leq \liminf _{n \rightarrow \infty} \varepsilon_{n} \log \mathbb{P}\left(W_{n} \in A\right) \\
& \leq \limsup _{n \rightarrow \infty} \varepsilon_{n} \log \mathbb{P}\left(W_{n} \in A\right) \leq-\inf \{\mathrm{I}(u): u \in \bar{A}\}
\end{aligned}
$$

where $\stackrel{\circ}{A}$ (resp. $\bar{A}$ ) denotes the interior (resp. closure) of $A$ in $\mathbb{R}^{p}$. This will denoted by $\left(W_{n}\right) \in \operatorname{LDP}\left(\varepsilon_{n}, \mathrm{I}\right)$ in the sequel. We refer to [7] for general informations about large deviation theory.

Theorem 2.2. Assume that (H.1)-(H.6) hold for some $\varepsilon_{n} \downarrow$, that (C.1) holds for $\widehat{c}_{1, n}$ and that (C.2) holds for $\widehat{c}_{2, n}$. Then, for all $\left\{x_{1}, \ldots, x_{p}\right\} \subset F$ such that $\Sigma_{\left(x_{1}, \ldots, x_{p}\right)}$ is regular,

$$
\left\{\varepsilon_{n}^{1 / 2} \kappa_{n}\left(x_{j}\right)^{-1} n \widehat{c}_{2, n}\left(x_{j}\right)\left(\widehat{f}_{n}\left(x_{j}, \widehat{c}_{1, n}\right)-f\left(x_{j}\right)\right): 1 \leq j \leq p\right\} \in \operatorname{LDP}\left(\varepsilon_{n}, \mathrm{I}_{\left(x_{1}, \ldots x_{p}\right)}\right)
$$

where

$$
\mathrm{I}_{\left(x_{1}, \ldots x_{p}\right)}: u \in \mathbb{R}^{p} \mapsto 2^{-1 t} u \Sigma_{\left(x_{1}, \ldots x_{p}\right)}^{-1} u .
$$

Let us mention some applications of Theorem 2.2. First, it entails that for all $s>0$ and all $x \in F$,

$$
\mathbb{P}\left(\varepsilon_{n}^{1 / 2} n\left|\kappa_{n}(x)^{-1} \widehat{c}_{2, n}(x)\left(\widehat{f}_{n}\left(x, \widehat{c}_{1, n}\right)-f(x)\right)\right| \geq s\right) \simeq \exp \left(-2^{-1} s^{2} \varepsilon_{n}^{-1}\right)
$$

(here $u_{n} \simeq v_{n}$ means that $\log u_{n} / \log v_{n} \rightarrow 1$ as $n \rightarrow \infty$ ). This fact and the Borel-Cantelli Lemma can be used to prove that under (H.1)-(H.6) with $\varepsilon_{n}=\log (n)^{-1}$, we have, for all $x \in F$,

$$
\limsup _{n \rightarrow \infty}\left((2 \log (n))^{1 / 2} \kappa_{n}(x)\right)^{-1} n \widehat{c}_{2, n}(x)\left|\widehat{f}_{n}\left(x, \widehat{c}_{1, n}\right)-f(x)\right| \leq 1 \text { a.s. }
$$

Moreover, it is well known that moderate deviation principle is a key tool to prove laws of the iterated logarithm (see e.g. [5] Th. 1-4-1). In terms of confidence intervals, Theorem 2.2 can also be useful to compute the logarithmic asymptotic level of confidence intervals with asymptotic level 0 . More precisely, for fixed $x \in E$ and $t>0$, consider the interval

$$
\mathbb{I}_{n}(x, t)=\left[\widehat{f}_{n}\left(x, \widehat{c}_{1, n}\right)-t \varepsilon_{n}^{-1 / 2} \kappa_{n}(x)\left(n \widehat{c}_{2, n}(x)\right)^{-1}, \widehat{f}_{n}\left(x, \widehat{c}_{1, n}\right)+t \varepsilon_{n}^{-1 / 2} \kappa_{n}(x)\left(n \widehat{c}_{2, n}(x)\right)^{-1}\right]
$$

where $\varepsilon_{n}=o(1)$. Then, Theorem 2.1 entails that

$$
\mathbb{P}\left(f(x) \notin \mathbb{I}_{n}(x, t)\right) \rightarrow 0 \text { as } n \rightarrow \infty .
$$


Now, if conditions of Theorem 2.2 hold for $\left(\varepsilon_{n}\right)$ we readily obtain that

$$
\limsup _{n \rightarrow \infty} \varepsilon_{n} \log \mathbb{P}\left(f(x) \notin \mathbb{I}_{n}(x, t)\right)=-2^{-1} t^{2}
$$

In other words, $\left(\mathbb{I}_{n}(x, t)\right)_{n \geq 1}$ is a sequence of confidence intervals for $f(x)$ with logarithmic level $2^{-1} t^{2}$ and speed $\left(\varepsilon_{n}\right)$ (see [29] Def. 1).

Finally, in estimation theory, Theorem 2.2 can be used to compute the Kallenberg efficiency of $\widehat{f}_{n}$ (see [22,23]).

\subsection{Estimation of $\mathbf{c}$}

At this stage, we present two estimates of $f$ which belong to our general class (6) with some particular estimations of $c$.

The first example has been previously introduced and studied in term of central limit theorem in case $(\mathrm{P})$ in $[13]$ and is defined by

$$
\widehat{f}_{n}^{\mathrm{loc}}(x)=\sum_{r=1}^{k_{n}} \kappa_{n, r}(x) \nu_{n, r} Y_{n, r}^{*}\left(1+N_{n, r}^{-1}\right) .
$$

It is readily seen that $\widehat{f}_{n}^{\text {loc }}$ can be written as in (6) with the localized estimator of $c$ :

$$
\widehat{c}_{n}(x)=\widehat{c}_{n}^{\mathrm{loc}}(x):=k_{n} \bar{\kappa}_{n}(x)\left(n \sum_{r=1}^{k_{n}} \kappa_{n, r}(x) N_{n, r}^{-1} \nu_{n, r} Y_{n, r}^{*}\right)^{-1}
$$

where

$$
\bar{\kappa}_{n}(x):=k_{n}^{-1} \sum_{r=1}^{k_{n}} \kappa_{n, r}(x)
$$

Now, as $c$ is constant, it may appear interesting to use a more global estimation of it. To this aim, we consider the global estimator

$$
\widehat{c}_{n}^{\text {glo }}=k_{n}\left(n \sum_{r=1}^{k_{n}} \nu_{n, r} Y_{n, r}^{*} N_{n, r}^{-1}\right)^{-1}
$$

leading to

$$
\widehat{f}_{n}^{\text {glo }}(x):=\widehat{f}_{n}\left(x ; \widehat{c}_{n}^{\text {glo }}\right)=\sum_{r=1}^{k_{n}}\left(\kappa_{n, r}(x)+\bar{\kappa}_{n}(x) N_{n, r}^{-1}\right) \nu_{n, r} Y_{n, r}^{*} .
$$

The following corollary shows that Theorems 2.1 and 2.2 can be applied to $\widehat{f}_{n}^{\text {loc }}$ and $\widehat{f}_{n}^{\text {glo }}$.

Corollary 2.1. Theorems 2.1 and 2.2 hold when $\widehat{c}_{n, 1} \in\left\{\widehat{c}_{n}^{\text {loc }} ; \widehat{c}_{n}^{\text {glo }}\right\}$ and $\widehat{c}_{n, 2}=\widehat{c}_{n}^{\text {glo }}$.

Remark 2.1. For the limit theorems considered here, $\widehat{f}_{n}^{\text {loc }}$ and $\widehat{f}_{n}^{\text {glo }}$ are equivalent. Nethertheless, it can be shown that $\widehat{c}_{n}^{\text {glo }}$ is a better estimator of $c$ than $\widehat{c}_{n}^{\text {loc }}$ and therefore $\widehat{f}_{n}^{\text {glo }}$ may be prefered to $\widehat{f}_{n}^{\text {loc }}$. On the other hand, if the intensity (2) of the point process is not totally uniform, it is clear that $\hat{f}_{n}^{\text {loc }}$, which is based on a local estimation of $c$, is more robust than $\widehat{f}_{n}^{\text {glo }}$.

\section{Applichtions}

We first introduce a general class of kernel estimators which will be shown to satisfy our main results given in Theorems 2.1 and 2.2. Then, we focus on the particular cases of Parzen-Rosenblatt and Dirichlet kernels. 


\subsection{General kernel estimates}

Here, in order to smooth (4), a sequence $K_{n}: E \times E \rightarrow \mathbb{R}$, of general smoothing kernels is introduced. Conditions on this sequence will be imposed later. The general integrated kernel estimate is defined by

$$
\begin{aligned}
\widehat{f}_{n}\left(x ; \widehat{c}_{n}\right) & =\int_{E} K_{n}(x, t) \widehat{f}_{n}^{\circ}\left(t ; \widehat{c}_{n}\right) \nu(\mathrm{d} t) \\
& =\sum_{r=1}^{k_{n}}\left(\int_{I_{n, r}} K_{n}(x, t) \nu(\mathrm{d} t)\right)\left(Y_{n, r}^{*}+\left(n \widehat{c}_{n} \nu_{n, r}\right)^{-1}\right) .
\end{aligned}
$$

It appears that (7) is a particular case of (6) with $\kappa_{n, r}(x)=\nu_{n, r}^{-1} \int_{I_{n, r}} K_{n}(x, t) \nu(\mathrm{d} t)$. In the case where the calculation of this mean value is computationally expansive, it can be approximated by $K_{n}\left(x, x_{n, r}\right)$ for some $x_{n, r} \in I_{n, r}$, leading to the simplified estimate

$$
\widetilde{f}_{n}\left(x ; \widehat{c}_{n}\right)=\sum_{r=1}^{k_{n}} \nu_{n, r} K_{n}\left(x, x_{n, r}\right)\left(Y_{n, r}^{*}+\left(n \widehat{c}_{n} \nu_{n, r}\right)^{-1}\right),
$$

which is still a particular case of (6) with $\kappa_{n, r}(x)=K_{n}\left(x, x_{n, r}\right)$.

In order to introduce the assumptions needed on $K_{n}$, we set, for all $x \in E$,

and

$$
\begin{gathered}
\Gamma_{n, r}(x)=\sup \left\{K_{n}(x, t)-K_{n}(x, s):(s, t) \in I_{n, r} \times I_{n, r}\right\}, \\
\Xi_{n}(x)=k_{n}\left|\sum_{r=1}^{k_{n}} \int_{I_{n, r} \times I_{n, r}} K_{n}(x, t)(f(s)-f(t)) \nu(\mathrm{d} t) \nu(\mathrm{d} s)\right|,
\end{gathered}
$$

$$
\Psi_{n}(x)=\left|\int_{E} K_{n}(x, t) f(t) \nu(\mathrm{d} t)-f(x)\right| .
$$

For the sake of simplicity, assume that, for all $n \geq 1$, the partitions $\left\{I_{n, r}: 1 \leq r \leq k_{n}\right\}$ are such that $\nu_{n, r}=k_{n}^{-1}$ for all $r \leq k_{n}$. We also set

and, for all function $g: E \rightarrow \mathbb{R}$, we note

$$
\Delta_{n}=\max _{r \leq k_{n}}\left(M_{n, r}-m_{n, r}\right)
$$

$$
\|g\|_{p}=\left(\int_{E}|g(t)|^{p} \nu(\mathrm{d} t)\right)^{1 / p}, \text { and }\|g\|_{I}=\sup _{t \in I}|g(t)|(I \subset E) .
$$

In this context, the general assumptions (H.1) - (H.6) can be expressed as:

(H.1) $k_{n} \uparrow \infty$ and $n^{-1} k_{n} \max \left(\log (n), \varepsilon_{n}^{-1}\right) \rightarrow 0$ as $n \rightarrow \infty$.

(H.2) $0<m \leq M<+\infty$ and $n k_{n}^{-1} \Delta_{n} \rightarrow 0$ as $n \rightarrow \infty$.

(K.1) For all $n \geq 1, \int_{E \times E}\left|K_{n}(x, t)\right| \nu(\mathrm{d} x) \nu(\mathrm{d} t)<\infty$.

(K.2) For all $\left(x_{1}, x_{2}\right) \in F \times F$,

$$
\sum_{r=1}^{k_{n}} \Gamma_{n, r}\left(x_{1}\right) \int_{I_{n, r}}\left|K_{n}\left(x_{2}, t\right)\right| \nu(\mathrm{d} t)=o\left(\left\|K_{n}\left(x_{1}, .\right)\right\|_{2}\left\|K_{n}\left(x_{2}, .\right)\right\|_{2}\right) \text { as } n \rightarrow \infty .
$$

(K.3) For all $\left(x_{1}, x_{2}\right) \in F \times F$,

$$
\left\langle K_{n}\left(x_{1}, .\right), K_{n}\left(x_{2}, .\right)\right\rangle_{2}\left(\left\|K_{n}\left(x_{1}, .\right)\right\|_{2}\left\|K_{n}\left(x_{2}, .\right)\right\|_{2}\right)^{-1} \rightarrow \sigma\left(x_{1}, x_{2}\right) \text { as } n \rightarrow \infty .
$$


(K.4) For all $x \in F$,

$$
\left(\varepsilon_{n} k_{n}\right)^{-1 / 2}\left\|K_{n}(x, .)\right\|_{2}^{-1}\left\|K_{n}(x, .)\right\|_{E} \rightarrow 0 \text { as } n \rightarrow \infty .
$$

(K.5) For all $x \in F$,

$$
\varepsilon_{n}^{1 / 2} n k_{n}^{-1 / 2}\left\|K_{n}(x, .)\right\|_{2}^{-1} \max \left(\Psi_{n}(x) ; \Xi_{n}(x)\right) \rightarrow 0 \text { as } n \rightarrow \infty .
$$

(K.6) For all $x \in F$,

$$
\varepsilon_{n}^{1 / 4} n k_{n}^{-3 / 4}\left\|K_{n}(x, .)\right\|_{2}^{-1 / 2}\left\|K_{n}(x, .)\right\|_{1}^{1 / 2} \Delta_{n} \rightarrow 0 \text { as } n \rightarrow \infty .
$$

(K.7) For all $x \in F$,

$$
\varepsilon_{n}^{1 / 2} n k_{n}^{-1 / 2}\left\|K_{n}(x, .)\right\|_{2}^{-1}\left(\sum_{r=1}^{k_{n}} \int_{I_{n, r}}\left(K_{n}(x, t)-K_{n}\left(x, x_{n, r}\right)\right) \nu(\mathrm{d} t)\right) \rightarrow 0 \text { as } n \rightarrow \infty .
$$

In the following, we set

$$
v_{n}(x)=n k_{n}^{-1 / 2}\left\|K_{n}(x, .)\right\|_{2}^{-1}
$$

and

$$
\Sigma_{\left(x_{1}, \ldots x_{p}\right)}=\left[\sigma\left(x_{i}, x_{j}\right)\right]_{1 \leq i, j \leq p} .
$$

The results established in Section 2 yield:

Theorem 3.1. a) If (H.1), (H.2) and (K.1)-(K.6) hold with $\left(\varepsilon_{n}\right)_{n \geq 1} \equiv 1$, then, for all $\widehat{c}_{1, n}$ (resp. $\left.\widehat{c}_{2, n}\right)$ such that (C.1) (resp. (C.2)) hold and all $\left(x_{1}, \ldots x_{p}\right) \subset F$,

$$
\left\{\widehat{c}_{2, n}\left(x_{j}\right) v_{n}\left(x_{j}\right)\left(\widehat{f}_{n}\left(x_{j} ; \widehat{c}_{1, n}\right)-f\left(x_{j}\right)\right): 1 \leq j \leq p\right\} \underset{\mathcal{D}}{\rightarrow} N\left(0, \Sigma_{\left(x_{1}, \ldots, x_{p}\right)}\right) .
$$

b) If, moreover, (K.7) holds with $\left(\varepsilon_{n}\right)_{n \geq 1} \equiv 1$, then for all $\left\{x_{1}, \ldots, x_{p}\right\} \subset F$,

$$
\left\{\widehat{c}_{2, n}\left(x_{j}\right) v_{n}\left(x_{j}\right)\left(\widetilde{f}_{n}\left(x_{j} ; \widehat{c}_{1, n}\right)-f\left(x_{j}\right)\right): 1 \leq j \leq p\right\} \underset{\mathcal{D}}{\rightarrow} N\left(0, \Sigma_{\left(x_{1}, \ldots, x_{p}\right)}\right)
$$

Theorem 3.2. a) For all $\varepsilon_{n} \downarrow 0$ such that (H.1), (H.2) and (K.1)-(K.6) hold, for all $\widehat{c}_{1, n}$ (resp. $\widehat{c}_{2, n}$ ) such that (C.1) (resp. (C.2)) hold and all $\left(x_{1}, \ldots x_{p}\right) \subset F$ such that $\Sigma_{\left(x_{1}, \ldots, x_{p}\right)}$ is regular,

$$
\left\{\varepsilon_{n}^{1 / 2} \widehat{c}_{2, n}\left(x_{j}\right) v_{n}\left(x_{j}\right)\left(\widehat{f}_{n}\left(x_{j} ; \widehat{c}_{1, n}\right)-f\left(x_{j}\right)\right): 1 \leq j \leq p\right\} \in \operatorname{LDP}\left(\varepsilon_{n}, \mathrm{I}_{\left(x_{1}, \ldots x_{p}\right)}\right)
$$

with

$$
\mathrm{I}_{\left(x_{1}, \ldots, x_{p}\right)}: u \in \mathbb{R}^{p} \mapsto 2^{-1 t} u \Sigma_{\left(x_{1}, \ldots, x_{p}\right)}^{-1} u .
$$

b) If, moreover, (K.7) holds, then

$$
\left\{\varepsilon_{n}^{1 / 2} \widehat{c}_{2, n}\left(x_{j}\right) v_{n}\left(x_{j}\right)\left(\widetilde{f}_{n}\left(x_{j} ; \widehat{c}_{1, n}\right)-f\left(x_{j}\right)\right): 1 \leq j \leq p\right\} \in \operatorname{LDP}\left(\varepsilon_{n}, \mathrm{I}_{\left(x_{1}, \ldots x_{p}\right)}\right) .
$$

Some illustrations of this result are now provided.

Remark 3.1. In all the applications below involving $\widetilde{f}_{n}, x_{n, r}$ will be defined as the center of $I_{n, r}$. 


\subsection{Parzen kernel estimates}

Here, we take $E=[0,1]^{d}\left(d \in \mathbb{N}^{*}\right), \nu$ is the Lebesgue measure on $E$ and $\left\{I_{n, r}: 1 \leq r \leq k_{n}\right\}$ is an equidistant partition of $E$ such that $I_{n, r}=\prod_{j=1}^{d} J_{n, r, j}$ where the $J_{n, r, j}$ are intervals of $[0,1]$ of length $k_{n}^{-1 / d}$, leading to $\nu_{n, r}=k_{n}^{-1}$ for all $1 \leq r \leq k_{n}$.

The multivariate Parzen kernel estimate is defined by

$$
K_{n}^{P R}(x, t)=h_{n}^{-d} K\left(h_{n}^{-1}(x-t)\right),
$$

where $K: \mathbb{R}^{d} \rightarrow \mathbb{R}^{+}$is a Parzen-Rosenblatt kernel such that $K \in L^{2}\left(\mathbb{R}^{d}\right)$, and $\left(h_{n}\right)$ is a sequence of positive real numbers tending to zero (see e.g. [18]).

In a first time, we will assume that there exists $\alpha \in(0,1]$ such that:

(PR.1a) $f$ is $\alpha$-Lipschitz,

(PR.1b) $K$ is uniformly continuous except on a finite set $D \subset \mathbb{R}^{d}$ and $\int_{\mathbb{R}^{d}}\|u\|_{\mathbb{R}^{d}}^{\alpha} K(u) \mathrm{d} u<\infty$.

Denote by $I_{p}$ the identity matrix of $\mathbb{R}^{p}$. We are now in position to prove a central limit theorem and a moderate deviation principle for $\widehat{f}_{n}$.

Corollary 3.1. Assume that (PR.1) holds and

$$
\text { (i) } n^{-1} k_{n} \log (n)=o(1), \quad \text { (ii) } h_{n}^{d} k_{n} \rightarrow \infty \quad \text { and } \quad \text { (iii) } n k_{n}^{-1 / 2} h_{n}^{\alpha+d / 2}=o(1) \text {. }
$$

Then, (9) holds for $F=(0,1)^{d}, v_{n}=n h_{n}^{d / 2} k_{n}^{-1 / 2}$ and $\Sigma_{\left(x_{1}, \ldots, x_{p}\right)}=\|K\|_{2}^{2} I_{p}$.

The best rate of convergence is obtained for $h_{n}=n^{-\frac{1}{\alpha+d}}$ and $k_{n}=n^{\frac{d}{\alpha+d}} u_{n}^{2}$, where $u_{n} \rightarrow \infty$ arbitrarily slowly. In this case, $v_{n}=n^{\frac{\alpha}{\alpha+d}} u_{n}^{-1}$.

Corollary 3.2. Assume that (PR.1) holds and for some $\varepsilon_{n} \rightarrow 0$,

(i) $n^{-1} k_{n} \max \left(\log (n), \varepsilon_{n}^{-1}\right)=o(1)$,

(ii) $h_{n}^{d} k_{n} \varepsilon_{n} \rightarrow \infty$ and

(iii) $n k_{n}^{-1 / 2} h_{n}^{\alpha+d / 2} \varepsilon_{n}^{1 / 2}=o(1)$.

Then, (11) holds for $F=(0,1)^{d}, v_{n}=n h_{n}^{d / 2} k_{n}^{-1 / 2}$ and $\Sigma_{\left(x_{1}, \ldots, x_{p}\right)}=\|K\|_{2}^{2} I_{p}$.

In particular, if (13) holds for $\varepsilon_{n}=\log (n)^{-1}$, then for all, $x \in F$,

$$
\limsup _{n \rightarrow \infty}(2 \log (n))^{-1 / 2} n h_{n}^{d / 2} k_{n}^{-1 / 2} \widehat{c}_{2, n}(x)\left|\widehat{f}_{n}\left(x, \widehat{c}_{1, n}\right)-f(x)\right| \leq\|K\|_{2}^{2} \text { a.s. }
$$

Remark 3.2. a) Corollary 3.1 shows that, when $f$ is $\alpha$-lipschitzian, the speed of convergence of $\widehat{f}_{n}$ can be chosen arbitrarily close to the minimax speed $n^{-\frac{\alpha}{\alpha+d}}$ (see [17]).

b) In the case of Poisson point process with $d=1, \widetilde{f}_{n}$ with Parzen Rosenblatt kernel has been studied in [12]. In particular [12] Theorem 6-2, gives a central limit theorem for $\widetilde{f}_{n}$ with optimal rate $v_{n}=n^{\frac{\alpha}{\alpha+5 / 4}} u_{n}^{-1}$. Hence, from asymptotical point of view, $\widehat{f}_{n}$ is better than $\widetilde{f}_{n}$.

Since our approach involves regularization of Geffroy's estimates it is natural to study the case where $f$ is more regular than just $\alpha$-lipschitzian. In the following, for simplicity, we take $E=[0,1]$ and we only deal with the central limit theorem. Assume that:

(PR.2a) $f$ is in $C^{2}(E)$

(PR.2b), There exists a finite set $D$ such that

$$
K \text { is } C^{1} \text { on } D^{c}, K^{\prime} \in L^{1}(\mathbb{R}) \text { and } \int_{\mathbb{R}} u^{2} K(u) \mathrm{d} u<\infty .
$$


Corollary 3.3. a) If (PR.2) holds and

(i) $n^{-1} k_{n} \log (n)=o(1)$, (ii) $h_{n} k_{n} \rightarrow \infty$, (iii) $n k_{n}^{-7 / 4} h_{n}^{1 / 4}=o$ (1) and (iv) $n k_{n}^{-1 / 2} h_{n}^{5 / 2}=o(1)$,

then, (9) is true for $F=(0,1), v_{n}=n h_{n}^{1 / 2} k_{n}^{-1 / 2}$ and $\Sigma_{\left(x_{1}, \ldots, x_{p}\right)}=\|K\|_{2}^{2} I_{p}$.

The choices of $h_{n}=n^{-5 / 17}$ and $k_{n}=n^{9 / 17} u_{n}^{2}$, where $u_{n} \rightarrow \infty$ arbitrarily slowly, lead to $v_{n}=n^{10 / 17} u_{n}^{-1}$.

b) If moreover, $K$ is even, 1-Lipschitzian and $C^{2}$ on $D^{c}$ with $K^{\prime \prime} \in L^{1}(\mathbb{R})$, then, under (i)-(iv) and

(v) $n k_{n}^{-5 / 2} h_{n}^{-3 / 2}=o(1),(10)$ is true for $F, v_{n}$ and $\Sigma_{\left(x_{1}, \ldots, x_{p}\right)}$ defined as above.

The choices of $h_{n}=n^{-2 / 7}$ and $k_{n}=n^{4 / 7} u_{n}^{2}$, where $u_{n} \rightarrow \infty$ arbitrarily slowly, lead to $v_{n}=n^{4 / 7} u_{n}^{-1}$.

\subsection{Projection estimates: Dirichlet kernels}

Let $f \in L^{2}(E, \nu)$ and $\left(e_{j}\right)_{j \in \mathbb{N}}$ be an orthonormal basis of $L^{2}(E, \nu)$. The expansion of $f$ on this basis is

$$
f(x)=\sum_{j=0}^{\infty} a_{j} e_{j}(x), \quad x \in E,
$$

where each $a_{j}=\int_{E} e_{j}(t) f(t) \nu(\mathrm{d} t)$ can be estimated by

$$
\widehat{a}_{j, k_{n}}=\sum_{r=1}^{k_{n}}\left(\int_{I_{n, r}} e_{j}(t) \nu(\mathrm{d} t)\right)\left(Y_{n, r}^{*}+\left(n \widehat{c}_{n} \nu_{n, r}\right)^{-1}\right), \quad 1 \leq j \leq b_{n} .
$$

This leads to an estimation of $f(x)$ via:

$$
\widehat{f}_{n}\left(x ; \widehat{c}_{n}\right)=\sum_{j=0}^{l_{n}} \widehat{a}_{j, k_{n}} e_{j}(x)=\sum_{r=1}^{k_{n}}\left(\int_{I_{n, r}} K_{n}^{D}(x, t) \nu(\mathrm{d} t)\right)\left(Y_{n, r}^{*}+\left(n \widehat{c}_{n} \nu_{n, r}\right)^{-1}\right),
$$

where $\left(l_{n}\right)$ is a sequence of integers tending to infinity and $K_{n}^{D}$ the Dirichlet's kernel associated to the orthonormal basis $\left(e_{j}\right)_{j \in \mathbb{N}}$ defined by

$$
K_{n}^{D}(x, t)=\sum_{j=0}^{l_{n}} e_{j}(x) e_{j}(t), \quad(x, t) \in E^{2} .
$$

It appears that (15) is a particular case of (7t) with $K_{n}=K_{n}^{D}$. Of course, the, sometimes easier to handle, estimate

$$
\widetilde{f}_{n}\left(x ; \widehat{c}_{n}\right)=\sum_{r=1}^{k_{n}} \nu_{n, r} K_{n}^{D}\left(x, x_{n, r}\right)\left(Y_{n, r}^{*}+\left(n \widehat{c}_{n} \nu_{n, r}\right)^{-1}\right),
$$

can also be defined. Below, we focus on the trigonometric basis on $E=[0,1], \nu$ is the Lebesgue measure on $E$, and for all $1 \leq r \leq k_{n}, I_{n, r}$ are disjoint intervals of $[0,1]$ of length $k_{n}^{-1}$ (and therefore $\nu_{n, r}=1 / k_{n}$ ).

This basis is defined for $x \in[0,1]$ by

$$
e_{0}(x)=1, \quad e_{2 j-1}(x)=2^{1 / 2} \cos (2 j \pi x), \quad e_{2 j}(x)=2^{1 / 2} \sin (2 j \pi x), \quad j \geq 1 .
$$

It is easily seen in that case that the Dirichlet kernel is

$$
\begin{aligned}
K_{n}^{D}(x, t) & =\frac{\sin \left(\left(1+l_{n}\right) \pi(x-t)\right)}{\sin (\pi(x-t))} \quad \text { for } x \neq t \\
& =1+l_{n} \quad \text { if } x=t .
\end{aligned}
$$


Besides, we assume that: $f$ is in $C^{2}([0,1]), f(0)=f(1)$ and $f^{\prime}(0)=f^{\prime}(1)$. In particular,

$$
\Delta_{n}=O\left(k_{n}^{-1}\right) .
$$

Corollary 3.4. a) Assume that

$$
\begin{aligned}
& \text { (i) } n^{-1} k_{n} \log (n)=o(1), \quad(\text { ii }) k_{n}^{-1} l_{n} \log \left(l_{n}\right)=o(1), \quad(i i i) n k_{n}^{-1 / 2} l_{n}^{-2}=O(1), \\
& \text { (iv) } n k_{n}^{-5 / 2} l_{n}^{1 / 2} \log \left(l_{n}\right)=o(1) \quad \text { and } \quad(v) n l_{n}^{-1 / 4} k_{n}^{-7 / 4} \log \left(l_{n}\right)^{1 / 2}=o(1) .
\end{aligned}
$$

Then (9) holds for $F=[0,1], v_{n}=n\left(l_{n} k_{n}\right)^{-1 / 2}$ and $\Sigma_{\left(x_{1}, \ldots, x_{p}\right)}=I_{p}$.

In particular, the choices of $l_{n}=n^{10 / 27}$ and $k_{n}=n^{14 / 27} \log (n)^{2 / 7} u_{n}^{2}$, where $u_{n} \rightarrow \infty$ arbitrarily slowly, lead to $v_{n}=n^{5 / 9} \log (n)^{-1 / 7} u_{n}^{-1}$.

b) If (i)-(iii) and (iv') $n k_{n}^{-5 / 2} l_{n}^{3 / 2} \log \left(l_{n}\right)=o(1)$ are true, then (10) holds for $F$, $v_{n}$ and $\Sigma_{\left(x_{1}, \ldots, x_{p}\right)}$ defined as above.

The choices of $l_{n}=n^{8 / 23}$ and $k_{n}=n^{14 / 23} \log (n)^{2 / 5} u_{n}^{2}$, where $u_{n} \rightarrow \infty$ arbitrarily slowly, lead to $v_{n}=n^{12 / 23} \log (n)^{-1 / 5} u_{n}^{-1}$.

Remark 3.3. a) From the asymptotical point of view, $\widehat{f}_{n}$ is still better than $\widetilde{f}_{n}$. Nevertheless, since when $f$ is $C^{2}$, the minimax speed of convergence is $n^{-2 / 3}$ (see [17]), the above estimates are suboptimal.

b) In the case of Poisson point process, $\widetilde{f}_{n}$ with the trigonometric Dirichlet kernel has been introduced by Girard and Jacob [11]. In that context, they get a pointwise central limit theorem (see [11] Cor. 3) but their result is not sharp and Corollary 3.4 (b) constitutes a significant improvement since, instead of $n^{12 / 23} \log (n)^{-1 / 5} u_{n}^{-1}$, they obtain an "optimal" speed of convergence of $n^{2 / 5} \log (n)^{-1 / 5}(\log \log (n))^{\varepsilon}(\varepsilon>0)$.

\subsection{Concluding remarks}

In terms of applications, we have presented examples of integrated smoothed kernel estimates $\widehat{f}_{n}$ and of discrete smoothed kernel estimates $\widetilde{f}_{n}$. In all the cases considered, $\widehat{f}_{n}$ is strictly better than $\widetilde{f}_{n}$ from asymptotical point of view (i.e. (K.7) is not implied by the other assumptions of Ths. 3.1 and 3.2). When $f \in C^{2}$, Parzen kernel leads to better speed of convergence than trigonometric Dirichlet kernel but it does not reach the minimax speed.

Of course, other kernels are allowed, in particular wavelet kernels can be treated in the framework of Theorems 3.1 and 3.2. This is part of our future work. More generaly, the following problem should be of interest: given a class of function $\mathcal{F}$, and assuming that $f \in \mathcal{F}$, what is the best estimate of type ( 7 ) and in which case could we raise the minimax speed of convergence?

\section{Proofs}

\subsection{Proofs of the results of Section 2}

Let us first introduce some notations useful for the sequel. For all $1 \leq r \leq k_{n}$, we define:

- $\lambda_{n, r}=n c \nu_{n, r} m_{n, r}, \quad \Lambda_{n, r}=n c \nu_{n, r} M_{n, r}, \quad \mu_{n, r}=n c \nu_{n, r} f_{n, r}$,

- $b_{n, r}=b_{n, r}\left(\varepsilon_{n}\right)=\left(3 \mu_{n, r}^{-1}\left(\varepsilon_{n}^{-1 / 2}\left(n \nu_{n, r}\right)^{1 / 2}+\log \left(\nu_{n, r}^{-1}\right)\right)\right)^{1 / 2}, \quad b_{n}=b_{n}\left(\varepsilon_{n}\right)=\max _{r \leq k_{n}} b_{n, r}$,

- $B_{n, r}=B_{n, r}\left(\varepsilon_{n}\right)=\left\{\left|N_{n, r}-\mu_{n, r}\right| \leq b_{n, r} \mu_{n, r}\right\}, \quad B_{n}=B_{n}\left(\varepsilon_{n}\right)=\bigcap_{r \leq k_{n}} B_{n, r}$,

- $Z_{n, r}^{*}=n c \nu_{n, r} Y_{n, r}^{*}, \quad E_{n, r}=\mathbb{E}\left(Z_{n, r}^{*} \mid N_{n, r}\right), \quad Z_{n, r}^{\circ}=Z_{n, r}^{*}-E_{n, r}$,

- $\beta_{n, r}=N_{n, r}^{-1}\left(N_{n, r}+1\right) E_{n, r}-\mu_{n, r}, \quad \zeta_{n, r}=N_{n, r}^{-1}\left(N_{n, r}+1\right) Z_{n, r}^{\circ}$,

- $\Theta_{n}(x)=\sum_{r=1}^{k_{n}} w_{n, r}(x) \zeta_{n, r}$. 
Moreover $\mathcal{B}(n, p), \mathcal{P}(\lambda)$ and $\mathcal{M}\left(n ; p_{1}, \ldots, p_{k}\right)$ stand respectively for the binomial, Poisson and multinomial distribution.

The proofs of the main results are built as follows. First, we use the fact that

$$
\kappa_{n}^{-1} n c\left(\hat{f}_{n}^{\mathrm{loc}}-f\right)=\Theta_{n}+\rho_{n}
$$

where

$$
\rho_{n}=\sum_{r=1}^{k_{n}} w_{n, r} \beta_{n, r}+\kappa_{n}^{-1} n c\left(f_{n}-f\right),
$$

will be shown to be a remaining part which vanishes in terms of central limit theorem and of moderate deviation principle (see Lem. 4.5 below). Therefore, to get the intended result for $\hat{f}_{n}^{\text {loc }}$, it will be sufficient to consider the dominant part $\Theta_{n}$, which, as a sum of conditionally independent and centered random variables, will be studied thanks to general results of Section 5. In a second time, it will be proved that for all $\widehat{c}_{n}$ such that (C.1) holds, $\widehat{f}_{n}\left(. ; \widehat{c}_{n}\right)$ and $\widehat{f}_{n}^{\text {loc }}$ are equivalent in terms of central limit theorem and of moderate deviation principle.

The next lemma contains some useful technical results:

Lemma 4.1. Under assumptions (H.1) and (H.2):

a) For all $1 \leq r \leq k_{n}$, and all $t \in\left[0, M_{n, r}\right]$,

$$
\mathbb{P}\left(Y_{n, r}^{*} \leq t \mid N_{n, r}\right)=\left(\frac{\int_{I_{n, r}} \min (f, t) \mathrm{d} \nu}{\int_{I_{n, r}} f \mathrm{~d} \nu}\right)^{N_{n, r}} .
$$

In particular, for all $t \in\left[0, m_{n, r}\right]$,

$$
\mathbb{P}\left(Y_{n, r}^{*} \leq t \mid N_{n, r}\right)=\left(t f_{n, r}^{-1}\right)^{N_{n, r}}
$$

b) For all large $n$,

$$
\max _{1 \leq r \leq k_{n}}\left|\left(1-\left(m_{n, r} f_{n, r}^{-1}\right)^{N_{n, r}}\right)-\mu_{n, r}^{-1} N_{n, r}\left(\mu_{n, r}-\lambda_{n, r}\right)\right| \mathbf{1}_{B_{n}} \leq 2\left(n c \delta_{n}\right)^{2} \text { a.s. }
$$

c) For all large $n$,

$$
\max _{1 \leq r \leq k_{n}}\left(\int_{\lambda_{n, r}}^{\Lambda_{n, r}} \mathbb{P}\left(Z_{n, r}^{*}>s \mid N_{n, r}\right) \mathrm{d} s\right) \mathbf{1}_{B_{n}} \leq 2\left(n c \delta_{n}\right)^{2} \text { a.s. }
$$

d) For all large $n$,

$$
\max _{1 \leq r \leq k_{n}}\left|\beta_{n, r}\right| \mathbf{1}_{B_{n}} \leq 4\left(n c \delta_{n}\right)^{2} \text { a.s. }
$$

e) For all large $n$,

$$
\max _{1 \leq r \leq k_{n}}\left|\mu_{n, r}-E_{n, r}-1\right| \mathbf{1}_{B_{n}} \leq 4\left(n c \delta_{n}\right)^{2}+2\left(b_{n}+\left(m c n \nu_{n}\right)^{-1}\right) \text { a.s. }
$$

f) For all $n$ large enough, all $1 \leq r \leq k_{n}$, and $s \geq 0$ :

$$
\begin{aligned}
\mathbb{P}\left(\left|Z_{n, r}^{\circ}\right| \geq s \mid N_{n, r}\right) \mathbf{1}_{B_{n, r}}= & \left(\mu_{n, r}^{-1}\left(E_{n, r}-s\right)\right)^{N_{n, r}} \mathbf{1}_{\left[0, E_{n, r}\right]}(s) \mathbf{1}_{B_{n, r}} \\
& +\left(1-\left(\mu_{n, r}^{-1}\left(E_{n, r}+s\right)\right)^{N_{n, r}}\right) \mathbf{1}_{\left[0, \lambda_{n, r}-E_{n, r}\right]}(s) \mathbf{1}_{B_{n, r}} \\
& +\mathbb{P}\left(Z_{n, r}^{*}>s+E_{n, r} \mid N_{n, r}\right) \mathbf{1}_{\left[\lambda_{n, r}-E_{n, r}, \Lambda_{n, r}-E_{n, r}\right]}(s) \mathbf{1}_{B_{n, r}} .
\end{aligned}
$$


g) For all $n$ large enough, all $1 \leq r \leq k_{n}$, and $l \geq 0$ :

$$
\begin{aligned}
\mathbb{E}\left(\left|Z_{n, r}^{\circ}\right|^{l} \mid N_{n, r}\right) \mathbf{1}_{B_{n, r}}= & \left(\lambda_{n, r}-E_{n, r}\right)^{l} \mathbf{1}_{B_{n, r}} \\
& +l !\left(\mu_{n, r}^{-1} E_{n, r}\right)^{N_{n, r}} \frac{N_{n, r} ! E_{n, r}^{l}}{\left(N_{n, r}+l\right) !} \mathbf{1}_{B_{n, r}} \\
& -\int_{0}^{\lambda_{n, r}-E_{n, r}} l s^{l-1}\left(\mu_{n, r}^{-1}\left(E_{n, r}+s\right)\right)^{N_{n, r}} \mathrm{~d} s \mathbf{1}_{B_{n, r}} \\
& +\int_{\lambda_{n, r}-E_{n, r}}^{\Lambda_{n, r}-E_{n, r}} l s^{l-1} \mathbb{P}\left(Z_{n, r}^{*}>s+E_{n, r} \mid N_{n, r}\right) d s \mathbf{1}_{B_{n, r}} .
\end{aligned}
$$

Proof. a) Set

$$
N_{n, r}^{+}(t)=N_{n}\left(D_{n, r} \cap\left(I_{n, r} \times\left(t, M_{n, r}\right]\right)\right)
$$

Then

$$
\mathbb{P}\left(Y_{n, r}^{*} \leq t \mid N_{n, r}\right)=\mathbb{P}\left(N_{n, r}^{+}(t)=0 \mid N_{n, r}\right) .
$$

But, as easily seen, conditionally to $N_{n, r}$,

$$
N_{n, r}^{+}(t) \rightsquigarrow \mathcal{B}\left(N_{n, r}, 1-\frac{\int_{I_{n, r}} \min (f, t) \mathrm{d} \nu}{\int_{I_{n, r}} f \mathrm{~d} \nu}\right) .
$$

Hence, we get the intended result.

b) For all $u \in[0,1]$ and $\alpha \geq 2$, set $\varphi_{\alpha}(u)=(1-u)^{\alpha}-(1-\alpha u)$, then, the Taylor formula entails,

$$
0 \leq \varphi_{\alpha}(u) \leq(\alpha u)^{2}
$$

Hence, for all large $n$ and all $\omega \in B_{n}$,

$$
\begin{aligned}
& \max _{1 \leq r \leq k_{n}}\left|\left(1-\left(m_{n, r} f_{n, r}^{-1}\right)^{N_{n, r}}\right)-\mu_{n, r}^{-1} N_{n, r}\left(\mu_{n, r}-\lambda_{n, r}\right)\right|(\omega) \\
& =\max _{1 \leq r \leq k_{n}} \varphi_{N_{n, r}(\omega)}\left(\mu_{n, r}^{-1}\left(\mu_{n, r}-\lambda_{n, r}\right)\right) \\
& \leq \max _{1 \leq r \leq k_{n}}\left(N_{n, r}(\omega) \mu_{n, r}^{-1}\left(\mu_{n, r}-\lambda_{n, r}\right)\right)^{2} \\
& \leq\left(1+b_{n}\right)^{2}\left(n c \delta_{n}\right)^{2},
\end{aligned}
$$

where we have used the facts that

$$
\max _{1 \leq r \leq k_{n}}\left(\mu_{n, r}-\lambda_{n, r}\right) \leq n c \delta_{n}=o(1)
$$

and for all $\omega \in B_{n}$,

$$
\max _{1 \leq r \leq k_{n}} N_{n, r}(\omega) \mu_{n, r}^{-1} \leq 1+b_{n} .
$$

Finally, we get the intended result since, by (H.1),

$$
b_{n} \leq\left(3(c m)^{-1}\left(\left(n \nu_{n} \varepsilon_{n}\right)^{-1 / 2}+\left(n \nu_{n}\right)^{-1} \log \left(\nu_{n}^{-1}\right)\right)\right)^{1 / 2}=o(1)
$$


c) Using (b), we get that for all large $n$ and all $\omega \in B_{n}$,

$$
\begin{aligned}
& \max _{1 \leq r \leq k_{n}} n c \nu_{n, r}\left(\int_{m_{n, r}}^{M_{n, r}} \mathbb{P}\left(Y_{n, r}^{*}>t \mid N_{n, r}\right) \mathrm{d} t\right)(\omega) \\
& \leq \max _{1 \leq r \leq k_{n}} n c \nu_{n, r}\left(M_{n, r}-m_{n, r}\right) \mathbb{P}\left(Y_{n, r}^{*}>m_{n, r} \mid N_{n, r}\right)(\omega) \\
& \leq n c \delta_{n} \max _{1 \leq r \leq k_{n}}\left(1-\left(m_{n, r} f_{n, r}^{-1}\right)^{N_{n, r}(\omega)}\right) \\
& \leq n c \delta_{n} \max _{1 \leq r \leq k_{n}} \mu_{n, r}^{-1} N_{n, r}(\omega)\left(\mu_{n, r}-\lambda_{n, r}\right)+\left(1+b_{n}\right)^{2}\left(n c \delta_{n}\right)^{3} \\
& \leq\left(1+b_{n}\right)\left(n c \delta_{n}\right)^{2}+\left(1+b_{n}\right)^{2}\left(n c \delta_{n}\right)^{3} .
\end{aligned}
$$

d) Using (a), we get

$$
\begin{aligned}
n c \nu_{n, r} \int_{0}^{m_{n, r}} \mathbb{P}\left(Y_{n, r}^{*}>t \mid N_{n, r}\right) \mathrm{d} t \\
=n c \nu_{n, r} \int_{0}^{m_{n, r}}\left(1-\left(t f_{n, r}^{-1}\right)^{N_{n, r}}\right) \mathrm{d} t \\
=\mu_{n, r} N_{n, r}\left(N_{n, r}+1\right)^{-1}-\left(\mu_{n, r}-\lambda_{n, r}\right) N_{n, r}\left(N_{n, r}+1\right)^{-1} \\
\quad+\lambda_{n, r}\left(N_{n, r}+1\right)^{-1}\left(1-\left(m_{n, r} f_{n, r}^{-1}\right)^{N_{n, r}}\right) \\
=\mu_{n, r} N_{n, r}\left(N_{n, r}+1\right)^{-1}-\mu_{n, r}^{-1}\left(\mu_{n, r}-\lambda_{n, r}\right)^{2} N_{n, r}\left(N_{n, r}+1\right)^{-1} \\
\quad+\lambda_{n, r}\left(N_{n, r}+1\right)^{-1}\left[\left(1-\left(m_{n, r} f_{n, r}^{-1}\right)^{N_{n, r}}\right)-\mu_{n, r}^{-1} N_{n, r}\left(\mu_{n, r}-\lambda_{n, r}\right)\right] .
\end{aligned}
$$

Hence, by (b), for all large $n$ and all $\omega \in B_{n}$,

$$
\begin{aligned}
& \max _{1 \leq r \leq k_{n}}\left|n c \nu_{n, r} \int_{0}^{m_{n, r}} \mathbb{P}\left(Y_{n, r}^{*}>t \mid N_{n, r}\right) \mathrm{d} t-\mu_{n, r} N_{n, r}\left(N_{n, r}+1\right)^{-1}\right|(\omega) \\
& \leq\left(\left(m c n \nu_{n}\right)^{-1}+\left(1-b_{n}\right)\left(1+b_{n}\right)^{2}\right)\left(n c \delta_{n}\right)^{2} \\
& \leq 2\left(n c \delta_{n}\right)^{2}
\end{aligned}
$$

which, combined with (c) and the fact that

$$
E_{n, r}=n c \nu_{n, r} \int_{0}^{M_{n, r}} \mathbb{P}\left(Y_{n, r}^{*}>t \mid N_{n, r}\right) \mathrm{d} t
$$

gives the result.

e)

$$
\begin{aligned}
\max _{1 \leq r \leq k_{n}}\left|\mu_{n, r}-E_{n, r}-1\right| \mathbf{1}_{B_{n}} & =\max _{1 \leq r \leq k_{n}}\left|\mu_{n, r}\left(N_{n, r}+1\right)^{-1}-1-\beta_{n, r}\left(1+N_{n, r}^{-1}\right)^{-1}\right| \mathbf{1}_{B_{n}} \\
& \leq \max _{1 \leq r \leq k_{n}}\left|\left(\mu_{n, r}-N_{n, r}\right)\left(N_{n, r}+1\right)^{-1}-\left(N_{n, r}+1\right)^{-1}-\beta_{n, r}\left(1+N_{n, r}^{-1}\right)^{-1}\right| \mathbf{1}_{B_{n}} \\
& \leq\left(1-b_{n}\right)^{-1}\left(b_{n}+\left(n c \nu_{n} m\right)^{-1}\right)+4\left(n c \delta_{n}\right)^{2} .
\end{aligned}
$$


f) By (21) and (e),

$$
\lim _{n \rightarrow \infty} \min _{1 \leq r \leq k_{n}} \min _{\omega \in B_{n}}\left(\lambda_{n, r}-E_{n, r}(\omega)\right)=1
$$

Hence, eventually,

$$
\min _{1 \leq r \leq k_{n}} \min _{\omega \in B_{n}}\left(\lambda_{n, r}-E_{n, r}(\omega)\right)>0
$$

and, for all large $n$, all $1 \leq r \leq k_{n}$, and $s \geq 0$ :

$$
\begin{aligned}
\mathbb{P} & \left(\left|Z_{n, r}^{*}-E_{n, r}\right| \geq s \mid N_{n, r}\right) \mathbf{1}_{B_{n}} \\
= & \mathbb{P}\left(Z_{n, r}^{*} \geq E_{n, r}+s \mid N_{n, r}\right) \mathbf{1}_{B_{n}}+\mathbb{P}\left(Z_{n, r}^{*} \geq E_{n, r}-s \mid N_{n, r}\right) \mathbf{1}_{B_{n}} \\
= & \left(\mu_{n, r}^{-1}\left(E_{n, r}-s\right)\right)^{N_{n, r}} \mathbf{1}_{\left[0, E_{n, r}\right]}(s) \mathbf{1}_{B_{n}} \\
& +\left(1-\left(\mu_{n, r}^{-1}\left(E_{n, r}+s\right)\right)^{N_{n, r}}\right) \mathbf{1}_{\left[0, \lambda_{n, r}-E_{n, r}\right]}(s) \mathbf{1}_{B_{n}} \\
& +\mathbb{P}\left(Z_{n, r}^{*}>s+E_{n, r}\right) \mathbf{1}_{\left[\lambda_{n, r}-E_{n, r}, \Lambda_{n, r}-E_{n, r}\right]}(s) \mathbf{1}_{B_{n}} .
\end{aligned}
$$

g) Follows from the fact that, by (e), for all $l \geq 1$ and $r \leq k_{n}$,

$$
\begin{aligned}
\mathbb{E}\left(\left|Z_{n, r}^{*}-E_{n, r}\right|^{l} \mid N_{n, r}\right)= & \int_{0}^{+\infty} l s^{l-1} \mathbb{P}\left(\left|Z_{n, r}^{*}-E_{n, r}\right|>s \mid N_{n, r}\right) \mathrm{d} s \\
= & \int_{0}^{E_{n, r}} l s^{l-1}\left(\mu_{n, r}^{-1}\left(E_{n, r}-s\right)\right)^{N_{n, r}} \mathrm{~d} s \\
& +\int_{0}^{\lambda_{n, r}-E_{n, r}} l s^{l-1}\left(1-\left(\mu_{n, r}^{-1}\left(E_{n, r}+s\right)\right)^{N_{n, r}}\right) \mathrm{d} s \\
& +\int_{\lambda_{n, r}-E_{n, r}}^{\Lambda_{n, r}-E_{n, r}} l s^{l-1} \mathbb{P}\left(Z_{n, r}^{*}>s+E_{n, r} \mid N_{n, r}\right) \mathrm{d} s
\end{aligned}
$$

and that

$$
\begin{aligned}
\int_{0}^{E_{n, r}} l s^{l-1}\left(\mu_{n, r}^{-1}\left(E_{n, r}-s\right)\right)^{N_{n, r}} \mathrm{~d} s & =\left(\mu_{n, r}^{-1} E_{n, r}\right)^{N_{n, r}} \int_{0}^{1} l u^{l-1}(1-u)^{N_{n, r}} \mathrm{~d} u \\
& =\left(\mu_{n, r}^{-1} E_{n, r}\right)^{N_{n, r}}\left(\left(N_{n, r}+l\right) !\right)^{-1} l ! N_{n, r} ! E_{n, r}^{l} .
\end{aligned}
$$

We are now in position to prove all we need to study the dominant term $\Theta_{n}$ :

Lemma 4.2. Under assumptions (H.1) and (H.2):

a) There exits a non negative real numbers $\eta_{n} \rightarrow 0$ such that, for all $n \geq 1$,

$$
\max _{1 \leq r \leq k_{n}}\left|\mathbb{E}\left(\zeta_{n, r}^{2} \mid N_{n, r}\right)-1\right| \mathbf{1}_{B_{n}} \leq \eta_{n} \text { a.s. }
$$

b)

$$
\max _{l \geq 1} \max _{1 \leq r \leq k_{n}} \mathbb{E}\left((l !)^{-1}\left|\zeta_{n, r}\right|^{l} \mid N_{n, r}\right)^{1 / l} \mathbf{1}_{B_{n}} \leq 4 \text { a.s. }
$$

c) For all $\left(\varepsilon_{n}\right)$ such that (H.1) holds,

$$
\limsup _{n \rightarrow \infty} \varepsilon_{n} \log \mathbb{P}\left(B_{n}^{c}\right)=-\infty
$$


Proof. a) Lemma 4.1 (g) for $l=2$ entails

$$
\begin{aligned}
\mathbb{E} & \left(Z_{n, r}^{\circ 2} \mid N_{n, r}\right) \mathbf{1}_{B_{n, r}} \\
= & \left(N_{n, r}+1\right)^{-2} \mu_{n, r}^{2} \mathbf{1}_{B_{n, r}}+\left(\left(\lambda_{n, r}-E_{n, r}\right)^{2}-\left(N_{n, r}+1\right)^{-2} \mu_{n, r}^{2}\right) \mathbf{1}_{B_{n, r}} \\
& +2\left(\mu_{n, r}^{-1} \lambda_{n, r}\right)^{N_{n, r}}\left(N_{n, r}+2\right)^{-1} \lambda_{n, r}\left(\left(N_{n, r}+1\right)^{-1}\left(N_{n, r}+2\right) E_{n, r}-\lambda_{n, r}\right) \mathbf{1}_{B_{n, r}} \\
& +2 \int_{\lambda_{n, r}-E_{n, r}}^{\Lambda_{n, r}-E_{n, r}} s \mathbb{P}\left(Z_{n, r}^{*}>s+E_{n, r} \mid N_{n, r}\right) \mathrm{d} s \mathbf{1}_{B_{n, r} .} .
\end{aligned}
$$

Now, by Lemma 4.1 (c) and (e),

$$
\begin{aligned}
& \max _{1 \leq r \leq k_{n}} \int_{\lambda_{n, r}-E_{n, r}}^{\Lambda_{n, r}-E_{n, r}} 2 s \mathbb{P}\left(Z_{n, r}^{*}>s+E_{n, r} \mid N_{n, r}\right) \mathrm{d} s \mathbf{1}_{B_{n, r}} \\
& \leq 2 \max _{1 \leq r \leq k_{n}}\left(\Lambda_{n, r}-E_{n, r}\right) \int_{\lambda_{n, r}}^{\Lambda_{n, r}} \mathbb{P}\left(Z_{n, r}^{*}>s \mid N_{n, r}\right) \mathrm{d} s \mathbf{1}_{B_{n, r}} \\
& \leq 8\left(n c \delta_{n}\right)^{2} \text { eventually. }
\end{aligned}
$$

By Lemma 4.1 (d), for all large $n$,

$$
\begin{aligned}
& \max _{1 \leq r \leq k_{n}}\left|\left(N_{n, r}+1\right)^{-1}\left(N_{n, r}+2\right) E_{n, r}-\lambda_{n, r}\right| \mathbf{1}_{B_{n, r}} \\
& =\max _{1 \leq r \leq k_{n}}\left|\left(N_{n, r}+1\right)^{-2} N_{n, r}\left(N_{n, r}+2\right) \beta_{n, r}+\left(\mu_{n, r}-\lambda_{n, r}\right)-\left(N_{n, r}+1\right)^{-2} \mu_{n, r}\right| \mathbf{1}_{B_{n, r}} \\
& \leq 4\left(n c \delta_{n}\right)^{2}+n c \delta_{n}+\left(1-b_{n}\right)^{-2}\left(n m c \nu_{n}\right)^{-1}=O\left(n \delta_{n}+\left(n \nu_{n}\right)^{-1}\right) .
\end{aligned}
$$

Furthermore, since $a^{2}-b^{2}=(a-b)^{2}+2(a-b) b$, and

$$
\begin{aligned}
& \max _{1 \leq r \leq k_{n}}\left|\lambda_{n, r}-E_{n, r}-\left(N_{n, r}+1\right)^{-1} \mu_{n, r}\right| \mathbf{1}_{B_{n, r}} \\
& =\max _{1 \leq r \leq k_{n}}\left|\left(N_{n, r}+1\right)^{-1} N_{n, r} \beta_{n, r}-\left(\mu_{n, r}-\lambda_{n, r}\right)\right| \mathbf{1}_{B_{n, r}} \\
& \leq 4\left(n c \delta_{n}\right)^{2}+n c \delta_{n} \text { eventually, }
\end{aligned}
$$

and we get that for all large $n$,

$$
\begin{aligned}
& \max _{1 \leq r \leq k_{n}}\left|\left(\lambda_{n, r}-E_{n, r}\right)^{2}-\left(\left(N_{n, r}+1\right)^{-1} \mu_{n, r}\right)^{2}\right| \mathbf{1}_{B_{n, r}} \\
& \leq\left(4\left(n c \delta_{n}\right)^{2}+n c \delta_{n}\right)^{2}+2\left(4\left(n c \delta_{n}\right)^{2}+n c \delta_{n}\right) \max _{1 \leq r \leq k_{n}}\left(N_{n, r}+1\right)^{-1} \mu_{n, r} \\
& \leq\left(4\left(n c \delta_{n}\right)^{2}+n c \delta_{n}\right)\left(4\left(n c \delta_{n}\right)^{2}+n c \delta_{n}+2\left(1-b_{n}\right)^{-1}\right)^{2} \\
& =O\left(n \delta_{n}\right) .
\end{aligned}
$$


Now, (23)-(26) lead to

$$
\begin{aligned}
\max _{1 \leq r \leq k_{n}}\left|\mathbb{E}\left(\zeta_{n, r}^{2} \mid N_{n, r}\right)-N_{n, r}^{-2} \mu_{n, r}^{2}\right| \mathbf{1}_{B_{n}} & =\max _{1 \leq r \leq k_{n}}\left(1+N_{n, r}^{-1}\right)^{2}\left|\mathbb{E}\left(Z_{n, r}^{\circ 2} \mid N_{n, r}\right)-\left(N_{n, r}+1\right)^{-2} \mu_{n, r}^{2}\right| \mathbf{1}_{B_{n}} \\
& \leq 4 \max _{1 \leq r \leq k_{n}}\left|\mathbb{E}\left(Z_{n, r}^{\circ 2} \mid N_{n, r}\right)-\left(N_{n, r}+1\right)^{-2} \mu_{n, r}^{2}\right| \mathbf{1}_{B_{n}} \\
& =O\left(n \delta_{n}+\left(n \nu_{n}\right)^{-1}\right)=o(1)
\end{aligned}
$$

And we get the result by (27) and the fact that,

$$
\max _{1 \leq r \leq k_{n}}\left|N_{n, r}^{-2} \mu_{n, r}^{2}-1\right| \mathbf{1}_{B_{n}} \leq\left(1-b_{n}\right)^{-2}\left(2+b_{n}\right) b_{n}=o(1) .
$$

b) Lemma 4.1 (g) and (22) entail that, for all large $n$, all $1 \leq r \leq k_{n}$, and all $l \geq 1$,

$$
\begin{aligned}
& \mathbb{E}\left((l !)^{-1}\left|Z_{n, r}^{*}-E_{n, r}\right|^{l} \mid N_{n, r}\right)^{1 / l} \mathbf{1}_{B_{n, r}} \\
& \leq\left(\left(N_{n, r}+1\right)^{-1} E_{n, r}+\left(\lambda_{n, r}-E_{n, r}\right)+\left(\Lambda_{n, r}-E_{n, r}\right)\right) \mathbf{1}_{B_{n, r}} \leq 4
\end{aligned}
$$

c) First we prove that, for all $n$ large enough and all $1 \leq r \leq k_{n}$,

$$
\mathbb{P}\left(B_{n, r}^{c}\right) \leq 2 \nu_{n, r} \exp \left(-\varepsilon_{n}^{-1 / 2}\left(n \nu_{n, r}\right)^{1 / 2}\right)
$$

In case $(\mathrm{E}), N_{n, r} \rightsquigarrow \mathcal{B}\left(n, n^{-1} \mu_{n, r}\right)$, Bernstein Inequality yields, for large $n$,

$$
\begin{aligned}
\mathbb{P}\left(\left|N_{n, r}-\mu_{n, r}\right|>b_{n, r} \mu_{n, r}\right) & \leq 2 \exp \left(-\frac{b_{n, r}^{2} \mu_{n, r}^{2}}{2 \mu_{n, r}+\frac{2}{3} b_{n, r} \mu_{n, r}}\right) \\
& \leq 2 \exp \left(-3^{-1} b_{n, r}^{2} \mu_{n, r}\right) .
\end{aligned}
$$

In case $(\mathrm{P}), N_{n, r} \rightsquigarrow \mathcal{P}\left(\mu_{n, r}\right)$, hence, using a classical inequality (see e.g. [31] p. 486), we get, for large $n$,

$$
\begin{aligned}
\mathbb{P}\left(\left|N_{n, r}-\mu_{n, r}\right|>b_{n, r} \mu_{n, r}\right) \leq & \exp \left(b_{n, r} \mu_{n, r}-\left(1+b_{n, r}\right) \mu_{n, r} \log \left(1+b_{n, r}\right)\right) \\
& +\exp \left(-b_{n, r} \mu_{n, r}-\left(1-b_{n, r}\right) \mu_{n, r} \log \left(1-b_{n, r}\right)\right) \\
\leq & \exp \left(-2^{-1}\left(1-b_{n, r}\right) b_{n, r}^{2} \mu_{n, r}\right)+\exp \left(-b_{n, r}^{2} \mu_{n, r}\right) \\
\leq & 2 \exp \left(-3^{-1} b_{n, r}^{2} \mu_{n, r}\right) .
\end{aligned}
$$

Hence we get the result since, by definition of $b_{n, r}$,

$$
\exp \left(-3^{-1} b_{n, r}^{2} \mu_{n, r}\right)=\nu_{n, r} \exp \left(-\varepsilon_{n}^{-1 / 2}\left(n \nu_{n, r}\right)^{1 / 2}\right)
$$

Now, by (28),

$$
\mathbb{P}\left(B_{n}^{c}\right) \leq \sum_{r \leq k_{n}} \mathbb{P}\left(B_{n, r}^{c}\right) \leq 2 \exp \left(-\varepsilon_{n}^{-1 / 2}\left(n \nu_{n}\right)^{1 / 2}\right)
$$

And, since $n \nu_{n} \varepsilon_{n} \rightarrow \infty$,

$$
\limsup _{n \rightarrow \infty} \varepsilon_{n} \log \mathbb{P}\left(B_{n}^{c}\right) \leq \limsup _{n \rightarrow \infty} \varepsilon_{n} \log (2)-\left(n \nu_{n} \varepsilon_{n}\right)^{1 / 2}=-\infty
$$


Corollary 4.1. a) Assume that (H.1)-(H.4) hold for $\left(\varepsilon_{n}\right)_{n \geq 1} \equiv 1$. Then, for all $\left\{x_{1}, \ldots, x_{p}\right\} \subset F$,

$$
\left(\Theta_{n}\left(x_{j}\right)\right)_{1 \leq j \leq p} \underset{\mathcal{D}}{\longrightarrow} N\left(0, \Sigma_{\left(x_{1}, \ldots x_{p}\right)}\right) .
$$

b) Assume that (H.1)-(H.4) hold for some $\varepsilon_{n} \rightarrow 0$. Then, for all $\left\{x_{1}, \ldots, x_{p}\right\} \subset F$ such that $\Sigma_{\left(x_{1}, \ldots, x_{p}\right)}$ is regular,

$$
\left(\varepsilon_{n}^{1 / 2} \Theta_{n}\left(x_{j}\right)\right)_{1 \leq j \leq p} \in \operatorname{LDP}\left(\varepsilon_{n}, \mathrm{I}_{\left(x_{1}, \ldots x_{p}\right)}\right) .
$$

Proof. Take $\left\{x_{1}, \ldots, x_{p}\right\} \subset F$ and set $W_{n, r}=\left(w_{n, r}\left(x_{j}\right)\right)_{1 \leq j \leq p} \in \mathbb{R}^{p}$. Since

$$
\left(\Theta_{n}\left(x_{j}\right)\right)_{1 \leq j \leq p}=\sum_{r=1}^{k_{n}} W_{n, r} \zeta_{n, r}
$$

(a) follows by Lemma 4.2 and Theorem 5.1 and (b) holds by Lemma 4.2 and Theorem 5.2.

The next lemma will be needed in the sequel:

Lemma 4.3. Let $\left(M_{r}\right)_{r \leq k} \rightsquigarrow \mathcal{M}\left(\phi ; p_{1}, \ldots, p_{k}\right)$ and $\left(\Pi_{r}\right)_{r \leq k} \rightsquigarrow \bigotimes_{r \leq k} \mathcal{P}\left(\phi p_{r}\right)$.

Then, for all $\left(a_{1}, \ldots, a_{k}\right) \in\left(\mathbb{R}^{+}\right)^{k}$ and $\eta>0$,

$$
\mathbb{P}\left(\sum_{r \leq k} a_{r} M_{r} \geq \eta\right) \leq 2 \mathbb{P}\left(\sum_{r \leq k} a_{r} \Pi_{r} \geq \eta\right) .
$$

Proof. Without loss of generality, we can assume that there exit i.i.d. r.v. $\left(\zeta_{i}\right)_{i \geq 1}$ and disjoint sets $\left(A_{r}\right)_{1 \leq r \leq k_{n}}$ such that $p_{r}=\mathbb{P}\left(\zeta_{1} \in A_{r}\right)$,

$$
M_{r}=\sharp\left\{i \leq \phi: \zeta_{i} \in A_{r}\right\} \text { and } \Pi_{r}=\sharp\left\{i \leq \Pi: \zeta_{i} \in A_{r}\right\},
$$

where $\Pi \rightsquigarrow \mathcal{P}(\phi)$ is independent of $\left(\zeta_{i}\right)_{i \geq 1}$. By independence of $\Pi$ and $\left(M_{r}\right)_{r \leq k}$,

$$
\mathbb{P}\left(\sum_{r \leq k} a_{r} M_{r} \geq \eta\right) \leq \mathbb{P}(\Pi<\phi) \mathbb{P}\left(\sum_{r \leq k} a_{r} M_{r} \geq \eta\right)+\mathbb{P}\left(\sum_{r \leq k} a_{r} \Pi_{r} \geq \eta\right) .
$$

Hence, since $\mathbb{P}(\Pi \geq \phi) \geq 1 / 2$ (see [1] Lemma 1 ), we get,

$$
\mathbb{P}\left(\sum_{r \leq k} a_{r} M_{r} \geq \eta\right) \leq \mathbb{P}(\Pi \geq \phi)^{-1} \mathbb{P}\left(\sum_{r \leq k} a_{r} \Pi_{r} \geq \eta\right) \leq 2 \mathbb{P}\left(\sum_{r \leq k} a_{r} \Pi_{r} \geq \eta\right) .
$$

The next lemma is useful to prove Corollary 2.1 (see also [14] and [28] for other applications).

Lemma 4.4. a) Assume that (H.1) and (H.2) hold for some $\left(\varepsilon_{n}\right)_{n \geq 1}$. Set $\left(u_{n, r}\right)_{r \leq k_{n}} \subset \mathbb{R}$ such that
(i) $\varepsilon_{n}^{1 / 2}\left(n \nu_{n}\right)^{-1}\left(n \delta_{n}\right)^{2} u_{n}^{(1)}=o(1)$,
(ii) $\varepsilon_{n}^{-1 / 2} u_{n}=o(1) \quad$ and
(iii) $\left(n \nu_{n}\right)^{-1} u_{n}^{(2)}=o(1)$,

with

$$
u_{n}:=\max _{1 \leq r \leq k_{n}}\left|u_{n, r}\right|, \quad u_{n}^{(1)}:=\sum_{r=1}^{k_{n}}\left|u_{n, r}\right| \quad \text { and } \quad u_{n}^{(2)}:=\sum_{r=1}^{k_{n}} u_{n, r}^{2} .
$$


Then, for all $\eta>0$,

$$
\limsup _{n \rightarrow \infty} \varepsilon_{n} \log \mathbb{P}\left(\varepsilon_{n}^{1 / 2}\left|\sum_{r=1}^{k_{n}} u_{n, r}\left(N_{n, r}^{-1} Z_{n, r}-1\right)\right| \geq \eta\right)=-\infty .
$$

b) (H.1), (H.2), (H.4) and (H.6), imply that (C.1) holds for $\widehat{c}_{n}^{\text {loc }}$ and $\widehat{c}_{n}^{\text {glo }}$.

c) (H.1) and (H.2) imply that (C.2) holds for $\widehat{c}_{n}^{\text {glo }}$.

Proof. a) By Lemma 4.2 (c) we just need to show that

$$
\limsup _{n \rightarrow \infty} \varepsilon_{n} \log \mathbb{P}\left(\varepsilon_{n}^{1 / 2}\left|\sum_{r=1}^{k_{n}} u_{n, r}\left(N_{n, r}^{-1} Z_{n, r}-1\right)\right| \mathbf{1}_{B_{n}} \geq \eta\right)=-\infty .
$$

Now,

$$
\left(N_{n, r}^{-1} Z_{n, r}-1\right) \mathbf{1}_{B_{n, r}}=N_{n, r}^{-1} Z_{n, r}^{\circ}+N_{n, r}^{-1} \beta_{n, r}+\chi_{n, r},
$$

with

$$
\chi_{n, r}:=\left(\mu_{n, r}\left(N_{n, r}+1\right)^{-1}-1\right) \mathbf{1}_{B_{n, r}} .
$$

Hence

$$
\begin{aligned}
& \varepsilon_{n}^{1 / 2}\left|\sum_{r=1}^{k_{n}} u_{n, r}\left(N_{n, r}^{-1} Z_{n, r}-1\right)\right| \mathbf{1}_{B_{n}} \\
& \leq \varepsilon_{n}^{1 / 2}\left|\sum_{r=1}^{k_{n}} u_{n, r} N_{n, r}^{-1} Z_{n, r}^{\circ}\right| \mathbf{1}_{B_{n}}+\varepsilon_{n}^{1 / 2}\left|\sum_{r=1}^{k_{n}} u_{n, r} N_{n, r}^{-1} \beta_{n, r}\right|+\varepsilon_{n}^{1 / 2}\left|\sum_{r=1}^{k_{n}} u_{n, r} \chi_{n, r}\right| .
\end{aligned}
$$

But, Lemma 4.1 (d) and by (i),

$$
\varepsilon_{n}^{1 / 2}\left|\sum_{r=1}^{k_{n}} u_{n, r} N_{n, r}^{-1} \beta_{n, r}\right|=O\left(\varepsilon_{n}^{1 / 2}\left(n \nu_{n}\right)^{-1}\left(n \delta_{n}\right)^{2} \sum_{r=1}^{k_{n}}\left|u_{n, r}\right|\right)=o(1) .
$$

Moreover, by Lemma 4.2 (b), the fact that, eventually,

$$
\max _{1 \leq r \leq k_{n}} N_{n, r}^{-1}\left|Z_{n, r}^{\circ}\right| \mathbf{1}_{B_{n}} \leq\left(1-b_{n}\right)^{-1}\left(1+m^{-1} \max _{1 \leq r \leq k_{n}}\left(M_{n, r}-m_{n, r}\right)\right) \leq 2,
$$

and (conditional) Bernstein inequality, we get that there exists $A>0$ such that, for all $\eta>0$,

$$
\begin{aligned}
& \mathbb{P}\left(\varepsilon_{n}^{1 / 2}\left|\sum_{r=1}^{k_{n}} u_{n, r} N_{n, r}^{-1} Z_{n, r}^{\circ}\right| \mathbf{1}_{B_{n}} \geq \eta \mid \mathcal{B}_{n}\right) \\
& \leq 2 \exp \left(-A \eta^{2} \varepsilon_{n}^{-1}\left(2 \sum_{r=1}^{k_{n}} u_{n, r}^{2} \mathbb{E}\left(Z_{n, r}^{\circ 2} \mid N_{n, r}\right) N_{n, r}^{-2} \mathbf{1}_{B_{n}}+2 \eta \varepsilon_{n}^{-1 / 2} u_{n}\right)^{-1}\right) \\
& \leq 2 \exp \left(-A \eta^{2} \varepsilon_{n}^{-1}\left(\left(m c n \nu_{n}\right)^{-2} u_{n}^{(2)}+2 \eta \varepsilon_{n}^{-1 / 2} u_{n}\right)^{-1}\right) .
\end{aligned}
$$


Therefore, by (ii) and (iii),

$$
\begin{aligned}
& \limsup _{n \rightarrow \infty} \varepsilon_{n} \log \mathbb{P}\left(\varepsilon_{n}^{1 / 2}\left|\sum_{r=1}^{k_{n}} u_{n, r} N_{n, r}^{-1} Z_{n, r}^{\circ}\right| \mathbf{1}_{B_{n}} \geq \eta\right) \\
& =\limsup _{n \rightarrow \infty} \varepsilon_{n} \log \mathbb{E}\left(\mathbb{P}\left(\varepsilon_{n}^{1 / 2}\left|\sum_{r=1}^{k_{n}} u_{n, r} N_{n, r}^{-1} Z_{n, r}^{\circ}\right| \mathbf{1}_{B_{n}} \geq \eta \mid \mathcal{B}_{n}\right)\right) \\
& \leq \log (2)-A \eta^{2} \liminf _{n \rightarrow \infty}\left(\left(m c n \nu_{n}\right)^{-2} u_{n}^{(2)}+2 \eta \varepsilon_{n}^{-1 / 2} u_{n}\right)^{-1}=-\infty
\end{aligned}
$$

and our last task is to show that

$$
\limsup _{n \rightarrow \infty} \varepsilon_{n} \log \mathbb{P}\left(\varepsilon_{n}^{1 / 2}\left|\sum_{r=1}^{k_{n}} u_{n, r} \chi_{n, r}\right| \geq \eta\right)=-\infty .
$$

a1) First, we consider case (P).

For all large $n$ and all $r \leq k_{n}$,

$$
\begin{aligned}
\left|\chi_{n, r}\right| & \leq\left(1-b_{n, r}\right)^{-1} \mu_{n, r}^{-1}\left(\left|N_{n, r}-\mu_{n, r}\right| \mathbf{1}_{B_{n, r}}+1\right) \\
& \leq\left(1-b_{n, r}\right)^{-1} b_{n, r}+\left(\left(1-b_{n, r}\right) \mu_{n, r}\right)^{-1} \leq 2 b_{n, r} .
\end{aligned}
$$

Hence, $\left(\chi_{n, r}-\mathbb{E}\left(\chi_{n, r}\right)\right)_{r \leq k_{n}}$ are independent, centered and eventually bounded by $4 b_{n}$. Moreover,

$$
\begin{aligned}
\mathbb{V}\left(\chi_{n, r}\right) & \leq\left(1-b_{n, r}\right)^{-2} \mu_{n, r}^{-2} \mathbb{E}\left(\left(\left|N_{n, r}-\mu_{n, r}\right|+1\right)^{2}\right) \\
& \leq\left(1-b_{n, r}\right)^{-2} \mu_{n, r}^{-2}\left(\mathbb{E}\left(\left(N_{n, r}-\mu_{n, r}\right)^{2}\right)^{1 / 2}+1\right)^{2} \\
& \leq\left(1-b_{n, r}\right)^{-2} \mu_{n, r}^{-2}\left(\mu_{n, r}^{1 / 2}+1\right)^{2} \\
& \leq\left(1-b_{n}\right)^{-2}\left(1+\left(m c n \nu_{n}\right)^{-1 / 2}\right)^{2}\left(m c n \nu_{n}\right)^{-1} \leq 2\left(m c n \nu_{n}\right)^{-1} \text { eventually. }
\end{aligned}
$$

Therefore, by Bernstein inequality, there exists $A>0$

$$
\begin{aligned}
\mathbb{P}\left(\varepsilon_{n}^{1 / 2}\left|\sum_{r=1}^{k_{n}} u_{n, r}\left(\chi_{n, r}-\mathbb{E}\left(\chi_{n, r}\right)\right)\right| \geq \eta\right) & \leq 2 \exp \left(-A \eta^{2} \varepsilon_{n}^{-1}\left(\sum_{r=1}^{k_{n}} u_{n, r}^{2} \mathbb{V}\left(\chi_{n, r}\right)+\eta \varepsilon_{n}^{-1 / 2} 4 b_{n} u_{n}\right)^{-1}\right) \\
& \leq 2 \exp \left(-A \eta^{2} \varepsilon_{n}^{-1}\left(2\left(m c n \nu_{n}\right)^{-1} u_{n}^{(2)}+4 \eta b_{n} \varepsilon_{n}^{-1 / 2} u_{n}\right)^{-1}\right),
\end{aligned}
$$

and (ii) and (iii) entail

$$
\limsup _{n \rightarrow \infty} \varepsilon_{n} \log \mathbb{P}\left(\varepsilon_{n}^{1 / 2}\left|\sum_{r=1}^{k_{n}} u_{n, r}\left(\chi_{n, r}-\mathbb{E}\left(\chi_{n, r}\right)\right)\right| \geq \eta\right)=-\infty
$$

Moreover,

$$
\left|\mathbb{E}\left(\left(\mu_{n, r}\left(N_{n, r}+1\right)^{-1}-1\right)\right)\right|=\mathrm{e}^{-\mu_{n, r}} \leq \exp \left(-m n c \nu_{n}\right)=o\left(n^{-1}\right)
$$


and, using (28), we get

$$
\left|\mathbb{E}\left(\left(\mu_{n, r}\left(N_{n, r}+1\right)^{-1}-1\right) \mathbf{1}_{B_{n, r}^{c}}\right)\right| \leq \mu_{n, r} \mathbb{P}\left(B_{n, r}^{c}\right)=n^{-1} O\left(\left(n \nu_{n}\right)^{2} \exp \left(-\varepsilon_{n}^{-1 / 2}\left(n \nu_{n}\right)^{1 / 2}\right)\right)=o\left(n^{-1}\right)
$$

thus,

$$
\max _{1 \leq r \leq k_{n}}\left|\mathbb{E}\left(\chi_{n, r}\right)\right|=o\left(n^{-1}\right)
$$

and

$$
\varepsilon_{n}^{1 / 2}\left|\sum_{r=1}^{k_{n}} u_{n, r} \mathbb{E}\left(\chi_{n, r}\right)\right|=o\left(\varepsilon_{n}^{1 / 2} n^{-1} \sum_{r=1}^{k_{n}}\left|u_{n, r}\right|\right)=o\left(\varepsilon_{n}^{1 / 2} n^{-1} k_{n} u_{n}\right)=o(1),
$$

which combined with (30) give the intended result.

a2) In case (E), we will use a coupling argument.

For all $n \geq 1$, define a sequence of i.i.d. random vectors $\left(\left(X_{n, i}^{\prime}, Y_{n, i}^{\prime}\right)\right)_{i \geq 1}$ with $\left(X_{n, i}^{\prime}, Y_{n, i}^{\prime}\right)_{i \leq n}=\left(X_{n, i}, Y_{n, i}\right)_{i \leq n}$.

Let $N_{n}^{\prime}(S) \rightsquigarrow \mathcal{P}(n)$, be independent of $\left(\left(X_{n, i}^{\prime}, Y_{n, i}^{\prime}\right)\right)_{i \geq 1}$, and consider the counting process

$$
N_{n}^{\prime}: D \in \mathcal{E} \otimes \mathcal{B}\left(\mathbb{R}^{+}\right) \mapsto \sharp\left\{i \leq N_{n}^{\prime}(S):\left(X_{n, i}^{\prime}, Y_{n, i}^{\prime}\right) \in D\right\}
$$

It is not hard to see that $N_{n}^{\prime}$ is a Poisson point process with intensity measure defined in (2). Now, set

$$
\begin{aligned}
& N_{n, r}^{\prime}=N_{n}^{\prime}\left(D_{n, r}\right), \quad B_{n, r}^{\prime}=\left\{\left|N_{n, r}^{\prime}-\mu_{n, r}\right| \leq b_{n, r} \mu_{n, r}\right\} \\
& \chi_{n, r}^{\prime}=\left(1-\mu_{n, r}\left(N_{n, r}^{\prime}+1\right)^{-1}\right) \mathbf{1}_{B_{n, r}^{\prime}} \text { and } B_{n}^{\prime}=\bigcap_{r \leq k_{n}} B_{n, r}^{\prime} .
\end{aligned}
$$

Since

$$
\left|\chi_{n, r}-\chi_{n, r}^{\prime}\right| \mathbf{1}_{B_{n} \cap B_{n}^{\prime}} \leq\left(1-b_{n}\right)^{-2} \mu_{n, r}^{-1}\left|N_{n, r}^{\prime}-N_{n, r}\right|
$$

we have, for all large $n$,

$$
\left|\sum_{r=1}^{k_{n}} u_{n, r} \chi_{n, r}\right| \mathbf{1}_{B_{n} \cap B_{n}^{\prime}} \leq\left|\sum_{r=1}^{k_{n}} u_{n, r} \chi_{n, r}^{\prime}\right|+2 \sum_{r=1}^{k_{n}}\left|u_{n, r}\right| \mu_{n, r}^{-1}\left|N_{n, r}^{\prime}-N_{n, r}\right| .
$$

By (a1),

$$
\limsup _{n \rightarrow \infty} \varepsilon_{n} \log \mathbb{P}\left(\varepsilon_{n}^{1 / 2}\left|\sum_{r=1}^{k_{n}} u_{n, r} \chi_{n, r}^{\prime}\right| \geq \eta\right)=-\infty .
$$

Moreover, since by Cauchy-Schwarz inequality and (iii),

$$
n^{-1 / 2} u_{n}^{(1)} \leq\left(n^{-1} \nu_{n}^{-1} u_{n}^{(2)}\right)^{1 / 2}=o(1)
$$

we can define a sequence $\left(\alpha_{n}\right)$ such that

$$
\alpha_{n} \uparrow \infty, \quad n^{-1 / 2} u_{n}^{(1)} \alpha_{n}=o(1) \quad \text { and } \quad \phi_{n}:=n^{1 / 2} \varepsilon_{n}^{-1 / 2} \alpha_{n} \in \mathbb{N} \text {. }
$$

Now, conditionally to $N_{n}^{\prime}(S)$,

$$
\left(\left|N_{n, r}^{\prime}-N_{n, r}\right|\right)_{1 \leq r \leq k_{n}} \rightsquigarrow \mathcal{M}\left(\left|N_{n}^{\prime}(S)-n\right| ; n^{-1} \mu_{n, 1}, \ldots, n^{-1} \mu_{n, k_{n}}\right),
$$


hence, Lemma 4.3 entails,

$$
\mathbb{P}\left(\varepsilon_{n}^{1 / 2} \sum_{r=1}^{k_{n}}\left|u_{n, r}\right| \mu_{n, r}^{-1}\left|N_{n, r}^{\prime}-N_{n, r}\right| \geq \eta\right) \leq 2 \mathbb{P}\left(\sum_{r=1}^{k_{n}} a_{n, r} \Pi_{n, r} \geq \eta\right)+\mathbb{P}\left(n^{-1 / 2} \varepsilon_{n}^{1 / 2}\left|N_{n}^{\prime}(S)-n\right| \geq \alpha_{n}\right),
$$

where

$$
a_{n, r}=\varepsilon_{n}^{1 / 2}\left|u_{n, r}\right| \mu_{n, r}^{-1}, \quad \text { and } \quad\left(\Pi_{n, r}\right)_{1 \leq r \leq k_{n}} \rightsquigarrow \bigotimes_{r \leq k_{n}} \mathcal{P}\left(\phi_{n} n^{-1} \mu_{n, r}\right) .
$$

Since $N_{n}^{\prime}(S)$ is a sum of $n$ i.i.d $\mathcal{P}(1)$ r.v. and $\alpha_{n} \uparrow \infty$, we have

$$
\limsup _{n \rightarrow \infty} \varepsilon_{n} \log \mathbb{P}\left(n^{-1 / 2} \varepsilon_{n}^{1 / 2}\left|N_{n}^{\prime}(S)-n\right| \geq \alpha_{n}\right)=-\infty
$$

(for $\left(\varepsilon_{n}\right)_{n \geq 1} \equiv 1$, this follows by the central limit theorem, for $\varepsilon_{n} \downarrow 0$ such that $n \varepsilon_{n} \rightarrow \infty$, it holds by [7] Th. 3-7-1). Moreover, for all large $n$,

$$
\begin{aligned}
\mathbb{P}\left(\sum_{r=1}^{k_{n}} a_{n, r} \Pi_{n, r} \geq \eta\right) & \leq \exp \left(-\eta \varepsilon_{n}^{-3 / 2} u_{n}^{-1}\right) \prod_{r=1}^{k_{n}} \mathbb{E}\left(\exp \left(\varepsilon_{n}^{-3 / 2} u_{n}^{-1} a_{n, r} \Pi_{n, r}\right)\right) \\
& =\exp \left(-\eta \varepsilon_{n}^{-3 / 2} u_{n}^{-1}+\phi_{n} n^{-1} \sum_{r=1}^{k_{n}} \mu_{n, r}\left(\exp \left(\varepsilon_{n}^{-1} u_{n}^{-1}\left|u_{n, r}\right| \mu_{n, r}^{-1}\right)-1\right)\right) \\
& \leq \exp \left(-\eta \varepsilon_{n}^{-3 / 2} u_{n}^{-1}+2 \phi_{n} n^{-1} \varepsilon_{n}^{-1} u_{n}^{-1} \sum_{r=1}^{k_{n}}\left|u_{n, r}\right|\right) \\
& =\exp \left(-\varepsilon_{n}^{-3 / 2} u_{n}^{-1}\left(\eta-2 \alpha_{n} n^{-1 / 2} u_{n}^{(1)}\right)\right) \leq \exp \left(-2^{-1} \eta \varepsilon_{n}^{-3 / 2} u_{n}^{-1}\right)
\end{aligned}
$$

where (35) follows from the facts that

$$
\max _{1 \leq r \leq k_{n}} \varepsilon_{n}^{-1} u_{n}^{-1}\left|u_{n, r}\right| \mu_{n, r}^{-1}=O\left(\left(n \nu_{n} \varepsilon_{n}\right)^{-1}\right)=o(1) \text { and } e^{t}-1 \leq 2 t \text { for small } t>0 .
$$

Consequently,

$$
\limsup _{n \rightarrow \infty} \varepsilon_{n} \log \mathbb{P}\left(\sum_{r=1}^{k_{n}} a_{n, r} \Pi_{n, r} \geq \eta\right) \leq-2^{-1} \eta \liminf _{n \rightarrow \infty} \varepsilon_{n}^{-1 / 2} u_{n}^{-1}=-\infty
$$

which, combined with (33) and (34) lead to

$$
\limsup _{n \rightarrow \infty} \varepsilon_{n} \log \mathbb{P}\left(\varepsilon_{n}^{1 / 2} \sum_{r=1}^{k_{n}}\left|u_{n, r}\right| \mu_{n, r}^{-1}\left|N_{n, r}^{\prime}-N_{n, r}\right| \geq \eta\right)=-\infty .
$$

Finally, Lemma 4.2 (c) yields

$$
\limsup _{n \rightarrow \infty} \varepsilon_{n} \log \mathbb{P}\left(B_{n}^{c} \cup B_{n}^{\prime c}\right)=-\infty
$$

and (29) follows by (31), (32), (36) and (37).

b) Note that, if $\widehat{c}_{n} \in\left\{\widehat{c}_{n}^{\text {loc }}, \widehat{c}_{n}^{\text {glo }}\right\}$, we can write

$$
\varepsilon_{n}^{1 / 2}\left|\sum_{r=1}^{k_{n}} w_{n, r}(x)\right|\left|\widehat{c}_{n}(x)^{-1}-c^{-1}\right|=\varepsilon_{n}^{1 / 2} c\left|\sum_{r=1}^{k_{n}} u_{n, r}(x)\left(N_{n, r}^{-1} Z_{n, r}^{*}-1\right)\right|,
$$

where $u_{n, r}(x)=w_{n, r}(x)$ for $\widehat{c}_{n}=\widehat{c}_{n}^{\text {loc }}$ and $u_{n, r}(x)=k_{n}^{-1}\left|\sum_{s=1}^{k_{n}} w_{n, s}(x)\right|$ for $\widehat{c}_{n}=\widehat{c}_{n}^{\text {glo }}$. 
In both cases, conditions (i) and (ii) of (a) hold respectively by (H.6) and (H.4) and condition (iii) is true by (H.1) since, here, $u_{n}^{(2)} \leq 1$. Hence, (a) shows that $\widehat{c}_{n}^{\text {loc }}$ and $\widehat{c}_{n}^{\text {glo }}$ satisfy (C.1).

c) Using the facts that for $a>0, b>0$, and $\eta>0$,

$$
|a-b| \geq \eta \Rightarrow\left|a b^{-1}-1\right| \geq \eta(a+\eta)^{-1}
$$

and that for all large $n$,

we get that, eventually,

$$
k_{n}^{-1}\left|\sum_{r=1}^{k_{n}}\left(N_{n, r}^{-1} Z_{n, r}^{*}-1\right)\right| \mathbf{1}_{B_{n}} \leq 2 b_{n}<\eta(c+\eta)^{-1}
$$

$$
\begin{aligned}
\mathbb{P}\left(\left|c-\widehat{c}_{n}^{\text {glo }}\right| \geq \eta\right) & \leq \mathbb{P}\left(k_{n}^{-1}\left|\sum_{r=1}^{k_{n}}\left(N_{n, r}^{-1} Z_{n, r}^{*}-1\right)\right| \geq \eta(c+\eta)^{-1}, B_{n}\right)+\mathbb{P}\left(B_{n}^{c}\right) \\
& \leq \mathbb{P}\left(B_{n}^{c}\right) .
\end{aligned}
$$

Hence, by Lemma 4.2 (c),

$$
\limsup _{n \rightarrow \infty} \varepsilon_{n} \log \mathbb{P}\left(\left|c-\widehat{c}_{n}^{\text {glo }}\right| \geq \eta\right) \leq \limsup _{n \rightarrow \infty} \varepsilon_{n} \log \mathbb{P}\left(B_{n}^{c}\right)=-\infty .
$$

Lemma 4.5. Assume that (H.1) - (H.6) hold. Then, for all $x \in F$ and any $\eta>0$,

$$
\limsup _{n \rightarrow \infty} \varepsilon_{n} \log \mathbb{P}\left(\varepsilon_{n}^{1 / 2}\left|\kappa_{n}(x)^{-1} n c\left(\widehat{f}_{n}\left(x, \widehat{c}_{n}\right)-f(x)\right)-\Theta_{n}(x)\right| \geq \eta\right)=-\infty .
$$

Proof. Since

$$
\begin{aligned}
\varepsilon_{n}^{1 / 2}\left|\kappa_{n}^{-1} n c\left(\hat{f}_{n}^{\text {loc }}-f\right)-\Theta_{n}\right| \mathbf{1}_{B_{n}} & \leq \varepsilon_{n}^{1 / 2} \sum_{r=1}^{k_{n}}\left|w_{n, r}\right|\left|\beta_{n, r}\right| \mathbf{1}_{B_{n}}+\kappa_{n}^{-1} n c\left|f_{n}-f\right| \\
& \leq 2 \varepsilon_{n}^{1 / 2}\left(n c \delta_{n}\right)^{2} \sum_{r=1}^{k_{n}}\left|w_{n, r}\right|+\varepsilon_{n}^{1 / 2} \kappa_{n}^{-1} n c\left|f_{n}-f\right| \\
& <2^{-1} \eta \text { eventually by (H.5) and (H.6) }
\end{aligned}
$$

we get that for large $n$,

$$
\limsup _{n \rightarrow \infty} \varepsilon_{n} \log \mathbb{P}\left(\varepsilon_{n}^{1 / 2}\left|\kappa_{n}^{-1} n c\left(\widehat{f}_{n}^{\text {loc }}-f\right)-\Theta_{n}\right| \geq 2^{-1} \eta\right) \leq \limsup _{n \rightarrow \infty} \varepsilon_{n} \log \mathbb{P}\left(B_{n}^{c}\right)=-\infty .
$$

Moreover, since

$$
\begin{aligned}
\mathbb{P}\left(\varepsilon_{n}^{1 / 2} \kappa_{n}^{-1} n c\left|\widehat{f}_{n}^{\text {loc }}-\widehat{f}_{n}\right| \geq 2^{-1} \eta\right)= & \mathbb{P}\left(c \varepsilon_{n}^{1 / 2}\left|\sum_{r=1}^{k_{n}} w_{n, r}\right|\left|\left(\widehat{c}_{n}^{\text {loc }}\right)^{-1}-\widehat{c}_{n}^{-1}\right| \geq 2^{-1} \eta\right) \\
\leq & \mathbb{P}\left(\varepsilon_{n}^{1 / 2}\left|\sum_{r=1}^{k_{n}} w_{n, r}\right|\left|\left(\widehat{c}_{n}^{\text {oc }}\right)^{-1}-c^{-1}\right| \geq 4^{-1} \eta c^{-1}\right) \\
& +\mathbb{P}\left(\varepsilon_{n}^{1 / 2}\left|\sum_{r=1}^{k_{n}} w_{n, r}\right|\left|\widehat{c}_{n}^{-1}-c^{-1}\right| \geq 4^{-1} \eta c^{-1}\right)
\end{aligned}
$$


Lemma 4.4 b), (C.1) and [7] Lemma 1-2-15 entail

$$
\limsup _{n \rightarrow \infty} \varepsilon_{n} \log \mathbb{P}\left(\varepsilon_{n}^{1 / 2} \kappa_{n}^{-1} n c\left|\hat{f}_{n}^{\text {loc }}-\widehat{f}_{n}\right| \geq 2^{-1} \eta\right)=-\infty
$$

Finally, since

$$
\begin{aligned}
& \mathbb{P}\left(\varepsilon_{n}^{1 / 2}\left|\kappa_{n}^{-1} n c\left(\widehat{f}_{n}-f\right)-\Theta_{n}\right| \geq \eta\right) \\
& \leq \mathbb{P}\left(\varepsilon_{n}^{1 / 2} \kappa_{n}^{-1} n c\left|\widehat{f}_{n}^{\text {loc }}-\widehat{f}_{n}\right| \geq 2^{-1} \eta\right)+\mathbb{P}\left(\varepsilon_{n}^{1 / 2}\left|\kappa_{n}^{-1} n c\left(\widehat{f}_{n}^{\text {loc }}-f\right)-\Theta_{n}\right| \geq 2^{-1} \eta\right),
\end{aligned}
$$

we get the intended result by (38), (39) and [7] Lemma 1-2-15.

Proof of Theorem 2.1. By Corollary 4.1 (a), Lemma 4.5 and [3] Theorem 4-1, we get that

$$
\left\{\kappa_{n}\left(x_{j}\right)^{-1} n c\left(\widehat{f}_{n}\left(x_{j}, \widehat{c}_{1, n}\right)-f\left(x_{j}\right)\right): 1 \leq j \leq p\right\} \underset{\mathcal{D}}{\rightarrow} N\left(0, \Sigma_{\left(x_{1}, \ldots x_{p}\right)}\right)
$$

which, combined with the fact that, $\widehat{c}_{2, n} \stackrel{\mathbb{P}}{\rightarrow} c$ by (C.2), gives the intended result.

Proof of Theorem 2.2. i) Corollary 4.1 (b), Lemma 4.5 and [7] Theorem 4-2-13, entail that the result hold for $\widehat{c}_{2, n}=c$.

ii) In the general case, for all $x \in F$, set

$$
D_{n}=n \kappa_{n}^{-1}\left(\widehat{c}_{2, n}-c\right)\left(\widehat{f}_{n}-f\right)
$$

By (i) and [7] Theorem 4-2-13, we just have to show that for all $x \in F$ and any $\eta>0$,

$$
\limsup _{n \rightarrow \infty} \varepsilon_{n} \log \left(\mathbb{P}\left(\varepsilon_{n}^{1 / 2}\left|D_{n}\right| \geq \eta\right)\right)=-\infty
$$

To this aim, note that, for all $\delta>0$,

$$
\mathbb{P}\left(\varepsilon_{n}^{1 / 2}\left|D_{n}\right| \geq \eta\right) \leq \mathbb{P}\left(\left|\widehat{c}_{2, n}-c\right|>\delta c\right)+\mathbb{P}\left(\varepsilon_{n}^{1 / 2} n \kappa_{n}^{-1} c\left|\widehat{f}_{n}\left(., \widehat{c}_{1, n}\right)-f\right| \geq \eta \delta^{-1}\right) .
$$

Therefore, by (C.2), [7] Lemma 1-2-15, and (i),

$$
\limsup _{n \rightarrow \infty} \varepsilon_{n} \log \left(\mathbb{P}\left(\varepsilon_{n}^{1 / 2}\left|D_{n}\right| \geq \eta\right)\right) \leq-2^{-1} \eta^{2} \delta^{-2}
$$

and we conclude by letting $\delta \downarrow 0$. 


\subsection{Proofs of the results of Section 3}

Proofs of Theorems 3.1 and 3.2. a) For all $x \in F$, we just verify (H.3)-(H.6) for $\kappa_{n, r}(x):=k_{n} \int_{I_{n, r}} K_{n}(x, t) \nu(\mathrm{d} t)$. By (K.2),

$$
\begin{aligned}
\sum_{r=1}^{k_{n}} \kappa_{n, r}\left(x_{1}\right) \kappa_{n, r}\left(x_{2}\right)= & k_{n}^{2} \sum_{r=1}^{k_{n}} \int_{I_{n, r}} \int_{I_{n, r}} K_{n}\left(x_{1}, s\right) K_{n}\left(x_{2}, t\right) \nu(\mathrm{d} s) \nu(\mathrm{d} t) \\
= & k_{n}\left\langle K_{n}\left(x_{1}, .\right), K_{n}\left(x_{2}, .\right)\right\rangle_{2} \\
& +k_{n}^{2} \sum_{r=1}^{k_{n}} \int_{I_{n, r}} \int_{I_{n, r}} K_{n}\left(x_{2}, t\right)\left(K_{n}\left(x_{1}, s\right)-K_{n}\left(x_{1}, t\right)\right) \nu(\mathrm{d} t) \nu(\mathrm{d} s) \\
= & k_{n}\left\langle K_{n}\left(x_{1}, .\right), K_{n}\left(x_{2}, .\right)\right\rangle_{2}+k_{n} O\left(\sum_{r=1}^{k_{n}} \Gamma_{n}\left(x_{1}\right) \int_{I_{n, r}}\left|K_{n}\left(x_{2}, t\right)\right| \nu(\mathrm{d} t)\right) \\
= & k_{n}\left[\left\langle K_{n}\left(x_{1}, .\right), K_{n}\left(x_{2}, .\right)\right\rangle_{2}+o\left(\left\|K_{n}\left(x_{1}, .\right)\right\|_{2}\left\|K_{n}\left(x_{2}, .\right)\right\|_{2}\right)\right] .
\end{aligned}
$$

Hence,

$$
\kappa_{n}(x)=k_{n}^{1 / 2}\left\|K_{n}(x, .)\right\|_{2}(1+o(1))
$$

and (K.3) entails,

$$
\sum_{r=1}^{k_{n}} w_{n, r}\left(x_{1}\right) w_{n, r}\left(x_{2}\right)=\sigma\left(x_{1}, x_{2}\right)+o(1),
$$

which is (H.3). Now, (40) entails for all large $n$,

$$
\begin{aligned}
\varepsilon_{n}^{-1 / 2} \max _{1 \leq r \leq k_{n}}\left|w_{n, r}(x)\right| & \leq 2\left(\varepsilon_{n} k_{n}\right)^{-1 / 2}\left\|K_{n}(x, .)\right\|_{2}^{-1} k_{n} \max _{1 \leq r \leq k_{n}}\left|\int_{I_{n, r}} K_{n}(t, x) \nu(\mathrm{d} t)\right| \\
& \leq 2\left(\varepsilon_{n} k_{n}\right)^{-1 / 2}\left\|K_{n}(x, .)\right\|_{2}^{-1}\left\|K_{n}(x, .)\right\|_{E}=o(1) \text { by (K.4), }
\end{aligned}
$$

i.e. (H.4) holds. In order to show (H.5), note that using (40) again, Fubini Theorem (which holds by (K.1)) and the triangle inequality yield

$$
\begin{aligned}
& \varepsilon_{n}^{1 / 2} n \kappa_{n}(x)^{-1}\left|\sum_{r=1}^{k_{n}} \nu_{n, r} \kappa_{n, r}(x) f_{n, r}-f(x)\right| \\
& =\varepsilon_{n}^{1 / 2} n \kappa_{n}(x)^{-1}\left|\sum_{r=1}^{k_{n}} k_{n} \int_{I_{n, r} \times I_{n, r}} K_{n}(x, t) f(s) \nu(\mathrm{d} t) \nu(\mathrm{d} s)-f(x)\right| \\
& \leq \varepsilon_{n}^{1 / 2} n \kappa_{n}(x)^{-1}\left(\Xi_{n}(x)+\Psi_{n}(x)\right) \\
& =O\left(\varepsilon_{n}^{1 / 2} n k_{n}^{-1 / 2}\left\|K_{n}(x, .)\right\|_{2}^{-1}\left(\Xi_{n}(x)+\Psi_{n}(x)\right)\right)=o(1) \quad \text { by (K.5). }
\end{aligned}
$$

Finally, we show that (H.6) holds.

$$
\begin{aligned}
\varepsilon_{n}^{1 / 2}\left(n \delta_{n}\right)^{2} \sum_{r=1}^{k_{n}}\left|w_{n, r}(x)\right| & =O\left(\varepsilon_{n}^{1 / 2}\left(n k_{n}^{-1} \Delta_{n}\right)^{2} k_{n}^{-1 / 2}\left\|K_{n}(x, .)\right\|_{2}^{-1} \sum_{r=1}^{k_{n}} k_{n}\left|\int_{I_{n, r}} K_{n}(t, x) \nu(\mathrm{d} t)\right|\right) \\
& =O\left(\varepsilon_{n}^{1 / 2} n^{2} k_{n}^{-3 / 2}\left\|K_{n}(x, .)\right\|_{2}^{-1}\left\|K_{n}(x, .)\right\|_{1} \Delta_{n}^{2}\right)=o(1) \text { by (K.6). }
\end{aligned}
$$


b) For all $x \in F$, it is easy to see that

$$
\begin{aligned}
\frac{n}{\kappa_{n}(x)}\left|\widehat{f}_{n}(x)-\widetilde{f}_{n}(x)\right| & \leq 4 M n k_{n}^{-1 / 2}\left\|K_{n}(x, .)\right\|_{2}^{-1}\left(\sum_{r=1}^{k_{n}} \int_{I_{n, r}}\left(K_{n}(x, t)-K_{n}\left(x, x_{n, r}\right)\right) \nu(\mathrm{d} t)\right) \\
& =o(1) \text { by }(\mathrm{K} .7),
\end{aligned}
$$

which, combined with (a), gives the intended result by standard arguments.

It is easy to see that, for all $x \in F$,

$$
\Xi_{n}(x) \leq\left\|K_{n}(x, .)\right\|_{1} \Delta_{n}
$$

Nevertheless, under regularity conditions, tighter bounds are possible for $\Xi_{n}(x)$ :

Lemma 4.6. Let $E=[0,1]^{d}\left(d \in \mathbb{N}^{*}\right)$, $\nu$ be the Lebesgue measure on $E$ and $\left\{I_{n, r}: 1 \leq r \leq k_{n}\right\}$ be a partition of $E$ such that $I_{n, r}=\prod_{j=1}^{d} J_{n, r, j}$ where the $J_{n, r, j}$ are interval of $[0,1]$ of length $k_{n}^{-1 / d}$. Assume that $f$ is in $C^{2}(E)$. Then,

$$
\Xi_{n}(x)=O\left(\left(\left\|K_{n}(x, .)\right\|_{1}+\sum_{r=1}^{k_{n}} \Gamma_{n, r}(x)\right) k_{n}^{-2 / d}\right) .
$$

Proof. Denote by $x_{n, r}$ the center of the cell $I_{n, r}$. Since $f$ is $C^{2}$, the multivariate Taylor formula yields:

$$
\begin{aligned}
\max _{r \leq k_{n}} \max _{s \in I_{n, r}}\left|f(s)-f\left(x_{n, r}\right)-\left\langle f^{\prime}\left(x_{n, r}\right), s-x_{n, r}\right\rangle_{\mathbb{R}^{d}}\right| & =O\left(\max _{r \leq k_{n}} \max _{s \in I_{n, r}}\left\|s-x_{n, r}\right\|_{\mathbb{R}^{d}}^{2}\right) \\
& =O\left(k_{n}^{-2 / d}\right) .
\end{aligned}
$$

Moreover,

Hence we get

$$
\int_{I_{n, r}}\left\langle f^{\prime}\left(x_{n, r}\right), s-x_{n, r}\right\rangle_{\mathbb{R}^{d}} \mathrm{~d} s=0
$$

Now, the triangle inequality entails

$$
\max _{r \leq k_{n}} \int_{I_{n, r}}\left(f(s)-f\left(x_{n, r}\right)\right) \mathrm{d} s=O\left(k_{n}^{-(d+2) / d}\right) .
$$

$$
\begin{aligned}
\Xi_{n}(x) \leq & k_{n}\left|\sum_{r=1}^{k_{n}} \int_{I_{n, r}} K_{n}(x, t)\left(\int_{I_{n, r}}\left(f(s)-f\left(x_{n, r}\right)\right) \mathrm{d} s\right) \mathrm{d} t\right| \\
& +\left|\sum_{r=1}^{k_{n}} \int_{I_{n, r}} K_{n}(x, t)\left(f(t)-f\left(x_{n, r}\right)\right) \mathrm{d} t\right| \\
= & O\left(\left\|K_{n}(x, .)\right\|_{1} k_{n}^{-2 / d}\right)+\left|\sum_{r=1}^{k_{n}} \int_{I_{n, r}} K_{n}(x, t)\left\langle f^{\prime}\left(x_{n, r}\right), t-x_{n, r}\right\rangle_{\mathbb{R}^{d}} \mathrm{~d} t\right| \\
= & O\left(\left\|K_{n}(x, .)\right\|_{1} k_{n}^{-2 / d}\right)+\left|\sum_{r=1}^{k_{n}} \int_{I_{n, r}}\left(K_{n}(x, t)-K_{n}\left(x, x_{n, r}\right)\right)\left\langle f^{\prime}\left(x_{n, r}\right), t-x_{n, r}\right\rangle_{\mathbb{R}^{d}} \mathrm{~d} t\right| \\
= & O\left(\left\|K_{n}(x, .)\right\|_{1} k_{n}^{-2 / d}+\sum_{r=1}^{k_{n}} \Gamma_{n, r}(x) \int_{I_{n, r}}\left\|t-x_{n, r}\right\|_{\mathbb{R}^{d}} \mathrm{~d} t\right) .
\end{aligned}
$$


Proof of Corollaries 3.1 and 3.2. We just verify the assumptions of Theorems 3.1 and 3.2 with $K_{n}=K_{n}^{P R}$. Set $x \in F$ and note that, by our assumptions on $f$ and $K$,

$$
\begin{gathered}
\Delta_{n}(x)=O\left(k_{n}^{-\alpha / d}\right), \quad \Xi_{n}(x)=O\left(k_{n}^{-\alpha / d}\right) \quad(\text { by }()), \\
\left\|K_{n}(x, .)\right\|_{1}=\int_{h_{n}^{-1}(x-E)} K(u) \mathrm{d} u=1+o(1) \\
\text { and }\left\|K_{n}(x, .)\right\|_{2}=h_{n}^{-\frac{d}{2}}\left(\int_{h_{n}^{-1}(x-E)} K^{2}(u) \mathrm{d} u\right)^{1 / 2}=h_{n}^{-\frac{d}{2}}\|K\|_{2}(1+o(1)) .
\end{gathered}
$$

Moreover, for $\varepsilon>0$ such that

$$
(t-\varepsilon, t+\varepsilon) \subset E,
$$

we have

$$
\begin{aligned}
\Psi_{n}(x)= & h_{n}^{-d}\left|\int_{E} K\left(h_{n}^{-1}(x-t)\right)(f(t)-f(x)) \mathrm{d} t\right| \\
= & \left|\int_{h_{n}^{-1}(x-E)} K(u)\left(f\left(x-h_{n} u\right)-f(x)\right) \mathrm{d} u\right| \\
\leq & \left|\int_{\|u\|_{\mathbb{R}^{d}} \leq \varepsilon h_{n}^{-1}} K(u)\left(f\left(x-h_{n} u\right)-f(x)\right) \mathrm{d} u\right| \\
& +\|f\|_{E}\left|\int_{\|u\|_{\mathbb{R}^{d}}>\varepsilon h_{n}^{-1}}\left(\varepsilon h_{n}^{-1}\right)^{-\alpha}\|u\|_{\mathbb{R}^{d}}^{\alpha} K(u) \mathrm{d} u\right| \\
= & O\left(h_{n}^{\alpha}\right) .
\end{aligned}
$$

To verify (H.2), note that

$$
\begin{aligned}
n k_{n}^{-1} \Delta_{n} & =O\left(n k_{n}^{-(1+\alpha / d)}\right) \\
& =O\left(\left(n k_{n}^{-1 / 2} h_{n}^{\alpha+d / 2} \varepsilon_{n}^{1 / 2}\right)\left(h_{n}^{d} k_{n} \varepsilon_{n}\right)^{-\left(\frac{1}{2}+\frac{\alpha}{d}\right)} \varepsilon_{n}^{\alpha / d}\right) \\
& =o(1) .
\end{aligned}
$$

(K.2) follows from the fact that

$$
\begin{aligned}
\sum_{r=1}^{k_{n}} \Gamma_{n, r}\left(x_{1}\right) \int_{I_{n, r}}\left|K_{n}\left(x_{2}, t\right)\right| \mathrm{d} t & \leq \max _{r \leq k_{n}} \Gamma_{n, r}\left(x_{1}\right) \\
& =O\left(h_{n}^{-d}\left(h_{n}^{d} k_{n}\right)^{-\alpha / d}\right)=o\left(h_{n}^{-d}\right)=o\left(\left\|K_{n}\left(x_{1}, .\right)\right\|_{2}\left\|K_{n}\left(x_{2}, .\right)\right\|_{2}\right) .
\end{aligned}
$$

To check (K.3), observe that, for all fixed $u \in \mathbb{R}^{d}$, and all $x_{1} \neq x_{2}$,

$$
K(u) K\left(u+h_{n}^{-1}\left(x_{1}-x_{2}\right)\right)=o(1) .
$$


Hence, by dominated convergence,

$$
\begin{aligned}
\left\langle K_{n}\left(x_{1}, .\right), K_{n}\left(x_{2}, .\right)\right\rangle_{2}\left(\left\|K_{n}\left(x_{1}, .\right)\right\|_{2}\left\|K_{n}\left(x_{2}, .\right)\right\|_{2}\right)^{-1} & \\
\quad & O\left(\int_{\mathbb{R}^{d}} K(u) K\left(u+h_{n}^{-1}\left(x_{1}-x_{2}\right)\right) \mathrm{d} u\right)=o(1) .
\end{aligned}
$$

For (K.4), note that,

$$
\varepsilon_{n}^{-1 / 2} k_{n}^{-1 / 2}\left\|K_{n}(x, .)\right\|_{2}^{-1}\left\|K_{n}(x, .)\right\|_{E}=\varepsilon_{n}^{-1 / 2} k_{n}^{-1 / 2} h_{n}^{-d / 2}\|K\|_{2}^{-1}\|K\|_{E}=o(1)
$$

with (ii). (K.5) follows by (iii) since

$$
n k_{n}^{-1 / 2} \varepsilon_{n}^{1 / 2}\left\|K_{n}(x, .)\right\|_{2}^{-1} \max \left(\Psi_{n}(x) ; \Xi_{n}(x)\right)=O\left(n k_{n}^{-1 / 2} h_{n}^{\alpha+d / 2} \varepsilon_{n}^{1 / 2}\right) .
$$

Finally, (K.6) follows by (ii) and (iii) since

$$
n k_{n}^{-3 / 4} \varepsilon_{n}^{1 / 4}\left\|K_{n}(x, .)\right\|_{2}^{-1 / 2}\left\|K_{n}(x, .)\right\|_{1}^{1 / 2} \Delta_{n}=\left(k_{n} \varepsilon_{n}\right)^{-1 / 4}\left(h_{n}^{d} k_{n}\right)^{-\alpha / d} O\left(n k_{n}^{-1 / 2} h_{n}^{\alpha+d / 2} \varepsilon_{n}^{1 / 2}\right) .
$$

Proof of Corollary 3.3. We just have to verify the assumptions of Theorem 3.1 with $K_{n}=K_{n}^{P R}, F=(0,1)$ and $\Sigma_{\left(x_{1}, \ldots, x_{p}\right)}=\|K\|_{2}^{2} I_{p}$.

For $h_{n}^{-1}(x-t) \in D^{c}$, denote by $K_{n}^{\prime}(x, t)$ and $K_{n}^{\prime \prime}(x, t)$ the first and second derivative of $K_{n}$ with respect to $t$. Set for all $x \in(0,1)$,

$$
R_{n}(x)=\left\{r \leq k_{n}: D \cap\left(h_{n}^{-1}\left(x-I_{n, r}\right)\right) \neq \emptyset\right\} .
$$

a) (K.1) holds trivialy and assumption (i) gives (H.1). To show (H.2), note that since $f$ is in $C^{2}(E),(i i)$ and (iii) yield

$$
n k_{n}^{-1} \Delta_{n}=O\left(n k_{n}^{-2}\right)=O\left(\left(n k_{n}^{-7 / 4} h_{n}^{1 / 4}\right)\left(k_{n} h_{n}\right)^{-1 / 4}\right)=o(1) .
$$

(K.2), (K.3) and (K.4) can be verified as in the proof of Corollary 3.1. We now consider (K.5). Observe that

$$
\begin{aligned}
\sum_{r=1}^{k_{n}} \Gamma_{n, r}(x) & =\sum_{r \in R_{n}(x)} \Gamma_{n, r}(x)+\sum_{r \notin R_{n}(x)} \Gamma_{n, r}(x) \\
& =\sharp R_{n}(x) O\left(h_{n}^{-1}\right)+\sum_{r \notin R_{n}(x)} \sup \left\{\left|\int_{s}^{t} K_{n}^{\prime}(x, u) \mathrm{d} u\right|:(s, t) \in I_{n, r} \times I_{n, r}\right\} \\
& \leq O\left(h_{n}^{-1}\right)+\int_{0}^{1}\left|K_{n}^{\prime}(x, u)\right| \mathrm{d} u=O\left(h_{n}^{-1}\right)+h_{n}^{-1} \int_{h_{n}^{-1}(x-1)}^{h_{n}^{-1} x}\left|K^{\prime}(t)\right| \mathrm{d} t \\
& =O\left(h_{n}^{-1}\right) .
\end{aligned}
$$

Hence, by (46, Lemma 4.6 and (43),

$$
\Xi_{n}(x)=O\left(h_{n}^{-1} k_{n}^{-2}\right) .
$$

Moreover, proceeding as in (45) and using Taylor formula with (14) we get,

$$
\begin{aligned}
\Psi_{n}(x) & \leq h_{n}^{2}\left\|f^{\prime \prime}\right\|_{E} \int_{|u| \leq \varepsilon h_{n}^{-1}} u^{2} K(u) \mathrm{d} u+h_{n}^{2} \varepsilon^{-2}\|f\|_{E} \int_{|u|>\varepsilon h_{n}^{-1}} u^{2} K(u) \mathrm{d} u \\
& =O\left(h_{n}^{2}\right) .
\end{aligned}
$$


Now, using (48) and (47) we obtain, by (iv),

$$
n k_{n}^{-1 / 2}\left\|K_{n}(x, .)\right\|_{2}^{-1} \max \left(\Psi_{n}(x) ; \Xi_{n}(x)\right)=O\left(n k_{n}^{-1 / 2} h_{n}^{1 / 2} \max \left(h_{n}^{2}, h_{n}^{-1} k_{n}^{-2}\right)\right)=o(1) .
$$

Finally, the fact that, by (iii),

$$
n k_{n}^{-3 / 4}\left\|K_{n}(x, .)\right\|_{2}^{-1 / 2}\left\|K_{n}(x, .)\right\|_{1}^{1 / 2} \Delta_{n} \leq n k_{n}^{-7 / 4} h_{n}^{1 / 4}=o(1),
$$

yields (K.6).

b) Since $K$ is 1 -Lipschitzian,

$$
\sum_{r \in R_{n}(x)}\left|\int_{I_{n, r}}\left(K_{n}(x, t)-K_{n}\left(x, x_{n, r}\right)\right) \mathrm{d} t\right|=\sharp R_{n}(x) O\left(\left(h_{n} k_{n}\right)^{-2}\right)=O\left(\left(h_{n} k_{n}\right)^{-2}\right) .
$$

Now, take $r \notin R_{n}(x)$. Using Taylor formula, we get that

$$
\begin{aligned}
\left|K_{n}(x, t)-K_{n}\left(x, x_{n, r}\right)-\left(t-x_{n, r}\right) K_{n}^{\prime}\left(x, x_{n, r}\right)\right| & =2^{-1}\left|\int_{\min \left(x_{n, r} ; t\right)}^{\max \left(x_{n, r} ; t\right)}\left(u-x_{n, r}\right) K_{n}^{\prime \prime}(x, u) \mathrm{d} u\right| \\
& \leq \int_{I_{n, r}}\left|u-x_{n, r}\right|\left|K_{n}^{\prime \prime}(x, u)\right| \mathrm{d} u \\
& \leq k_{n}^{-1} \int_{I_{n, r}}\left|K_{n}^{\prime \prime}(x, u)\right| \mathrm{d} u
\end{aligned}
$$

which, combined with the fact that $x_{n, r}$ is the center of $I_{n, r}$, leads to

$$
\begin{aligned}
\left|\int_{I_{n, r}}\left(K_{n}(x, t)-K_{n}\left(x, x_{n, r}\right)\right) \mathrm{d} t\right| \leq & \int_{I_{n, r}}\left|K_{n}(x, t)-K_{n}\left(x, x_{n, r}\right)-\left(t-x_{n, r}\right) K_{n}^{\prime}\left(x, x_{n, r}\right)\right| \mathrm{d} t \\
& +\left|K_{n}^{\prime}\left(x, x_{n, r}\right) \int_{I_{n, r}}\left(t-x_{n, r}\right) \mathrm{d} t\right| \\
\leq & k_{n}^{-2} \int_{I_{n, r}}\left|K_{n}^{\prime \prime}(x, u)\right| \mathrm{d} u
\end{aligned}
$$

and finally entails that,

$$
\begin{aligned}
\left|\sum_{r \notin R_{n}(x)} \int_{I_{n, r}}\left(K_{n}(x, t)-K_{n}\left(x, x_{n, r}\right)\right) \mathrm{d} t\right| & \leq k_{n}^{-2} \int_{E}\left|K_{n}^{\prime \prime}(x, u)\right| \mathrm{d} u \\
& =O\left(\left(h_{n} k_{n}\right)^{-2}\right)
\end{aligned}
$$

where we have used the fact that

$$
\begin{aligned}
\int_{E}\left|K_{n}^{\prime \prime}(x, u)\right| \mathrm{d} u & =h_{n}^{-3} \int_{E}\left|K^{\prime \prime}\left(h_{n}^{-1}(x-u)\right)\right| \mathrm{d} u \\
& =h_{n}^{-2} \int_{h_{n}^{-1}(x-E)}\left|K^{\prime \prime}(t)\right| \mathrm{d} t=O\left(h_{n}^{-2}\right) .
\end{aligned}
$$


Now, by (44), (49), (50), we obtain that

$$
\begin{aligned}
& n k_{n}^{-1 / 2}\left\|K_{n}(x, .)\right\|_{2}^{-1}\left|\sum_{r=1}^{k_{n}} \int_{I_{n, r}}\left(K_{n}(x, t)-K_{n}\left(x, x_{n, r}\right)\right) \mathrm{d} t\right|= \\
& O\left(n k_{n}^{-1 / 2} h_{n}^{1 / 2}\left(h_{n} k_{n}\right)^{-2}\right)=O\left(n k_{n}^{-5 / 2} h_{n}^{-3 / 2}\right),
\end{aligned}
$$

and (K.7) follows by (vi).

Proof of Corollary 3.4. We verify the assumptions of Theorem 3.1 with $K_{n}=K_{n}^{D}$. In what follows, the first and second derivative of $K_{n}(x, t)$ with respect to $t$ are denoted by $K_{n}^{\prime}(x, t)$ and $K_{n}^{\prime \prime}(x, t)$.

a) (K.1) holds trivialy. Assumptions (i) is (H.1). Since

$$
n k_{n}^{-2}=\left(n l_{n}^{-1 / 4} k_{n}^{-7 / 4}\right)\left(k_{n}^{-1} l_{n}\right)^{1 / 4}
$$

(H.2) follows by (ii) and (v). In order to verify (K.2)-(K.6) we will use the following well known facts (see e.g. [32])

$$
\begin{gathered}
\left\|K_{n}(x, .)\right\|_{E}=1+l_{n}, \quad\left\|K_{n}(x, .)\right\|_{2}=\left(1+l_{n}\right)^{1 / 2},\left\|K_{n}(x, .)\right\|_{1}=O\left(\log \left(l_{n}\right)\right), \\
\left\langle K_{n}\left(x_{1}, .\right), K_{n}\left(x_{2}, .\right)\right\rangle_{2}=K_{n}\left(x_{1}, x_{2}\right)=o\left(l_{n}\right) \text { for } x_{1} \neq x_{2}, \\
\int_{0}^{1}\left|K_{n}^{\prime}(x, t)\right| \mathrm{d} t=O\left(l_{n} \log \left(l_{n}\right)\right), \\
\int_{0}^{1}\left|K_{n}^{\prime \prime}(x, t)\right| \mathrm{d} t=O\left(l_{n}^{2} \log \left(l_{n}\right)\right) .
\end{gathered}
$$

Since $f$ is $C^{2}$, and taking into account of $f(0)=f(1)$ and $f^{\prime}(0)=f^{\prime}(1)$, a double integration by parts yields that there exists a bounded sequence $\left(\alpha_{j}\right)_{j \geq 1}$, such that

$$
\int_{0}^{1} f(t) e_{j}(t) \mathrm{d} t=\alpha_{j} j^{-2} \int_{0}^{1} f^{\prime \prime}(t) e_{j}(t) \mathrm{d} t
$$

Hence, using Cauchy Schwarz inequality, we get

$$
\begin{aligned}
\Psi_{n}(x) & =\left|\sum_{j>l_{n}} \alpha_{j} j^{-2} \int_{0}^{1} f^{\prime \prime}(t) e_{j}(t) \mathrm{d} t e_{j}(x)\right| \\
& =O\left(\sum_{j>l_{n}} j^{-4}\right)^{1 / 2}\left(\sum_{j>l_{n}}\left(\int_{0}^{1} f^{\prime \prime}(t) e_{j}(t) \mathrm{d} t\right)^{2}\right)^{1 / 2} \\
& =o\left(l_{n}^{-3 / 2}\right) .
\end{aligned}
$$

Since $\max _{j \geq 1} j^{-1}\left\|e_{j}^{\prime}\right\|_{E}=O(1)$, the Taylor formula gives

$$
\begin{aligned}
\max _{1 \leq r \leq k_{n}} \Gamma_{n, r}(x) & \leq \max _{1 \leq r \leq k_{n}} \sum_{j=0}^{l_{n}}\left|e_{j}(x)\right| \sup \left\{e_{j}(t)-e_{j}(s):(s, t) \in I_{n, r} \times I_{n, r}\right\} \\
& =O\left(k_{n}^{-1} \sum_{j=0}^{l_{n}} j\right)=O\left(k_{n}^{-1} l_{n}^{2}\right) .
\end{aligned}
$$


By (53), we get

$$
\begin{aligned}
\sum_{r=1}^{k_{n}} \Gamma_{n, r}(x) & =\sum_{r=1}^{k_{n}} \sup \left\{\left|\int_{s}^{t} K_{n}^{\prime}(x, u) \mathrm{d} u\right|:(s, t) \in I_{n, r} \times I_{n, r}\right\} \\
& \leq \int_{0}^{1}\left|K_{n}^{\prime}(x, u)\right| \mathrm{d} u=O\left(l_{n} \log \left(l_{n}\right)\right) .
\end{aligned}
$$

Therefore, Lemma 4.6 entails

$$
\Xi_{n}(x)=O\left(k_{n}^{-2} l_{n} \log \left(l_{n}\right)\right) .
$$

To verify (K.2), observe that by (51), (56) and (ii),

$$
\begin{aligned}
\sum_{r=1}^{k_{n}} \Gamma_{n, r}\left(x_{1}\right) \int_{I_{n, r}}\left|K_{n}\left(x_{2}, t\right)\right| \mathrm{d} t & \leq\left\|K_{n}\left(x_{2}, .\right)\right\|_{1} \max _{1 \leq r \leq k_{n}} \Gamma_{n, r}\left(x_{1}\right) \\
& =O\left(k_{n}^{-1} l_{n}^{2} \log \left(l_{n}\right)\right) \\
& =o\left(l_{n}\right)=o\left(\left\|K_{n}\left(x_{1}, .\right)\right\|_{2}\left\|K_{n}\left(x_{2}, .\right)\right\|_{2}\right) .
\end{aligned}
$$

(K.3) follows by (51) and (52). (K.4) holds by (51) and (ii).

In order to check (K.5), observe that (51), (55, (57), (iii) and (iv) yield

$$
n k_{n}^{-1 / 2}\left\|K_{n}(x, .)\right\|_{2}^{-1} \max \left(\Psi_{n}(x) ; \Xi_{n}(x)\right)=O\left(n k_{n}^{-1 / 2} l_{n}^{-1 / 2} \max \left(l_{n}^{-3 / 2} ; k_{n}^{-2} l_{n} \log \left(l_{n}\right)\right)\right)=o(1) .
$$

Finally, (51) and (v) give (K.6).

b) It is readily seen that (iv') implies (iv). Moreover, since

$$
n l_{n}^{-1 / 4} k_{n}^{-7 / 4} \log \left(l_{n}\right)^{1 / 2}=k_{n}^{-1 / 4}\left(n k_{n}^{-1 / 2} l_{n}^{-2}\right)^{1 / 2}\left(n k_{n}^{-5 / 2} l_{n}^{3 / 2} \log \left(l_{n}\right)\right)^{1 / 2}
$$

we get that (iv') and (iii) imply (v). Therefore (K.2)-(K.6) holds by a). To verify (K.7), note that, by Taylor formula, and since $x_{n, r}$ is the center of $I_{n, r}$, we get

$$
\begin{aligned}
\left|\int_{I_{n, r}}\left(K_{n}(x, t)-K_{n}\left(x, x_{n, r}\right)\right) \mathrm{d} t\right| & =2^{-1}\left|\int_{I_{n, r}}\left(\int_{\min \left(x_{n, r} ; t\right)}^{\max \left(x_{n, r} ; t\right)}\left(u-x_{n, r}\right) K_{n}^{\prime \prime}(x, u) \mathrm{d} u\right) \mathrm{d} t\right| \\
& \leq \int_{I_{n, r}}\left(\int_{I_{n, r}}\left|u-x_{n, r}\right|\left|K_{n}^{\prime \prime}(x, u)\right| \mathrm{d} u\right) \mathrm{d} t \\
& \leq k_{n}^{-2} \int_{I_{n, r}}\left|K_{n}^{\prime \prime}(x, u)\right| \mathrm{d} u .
\end{aligned}
$$

This and (54) entails

$$
\begin{aligned}
\left|\sum_{r=1}^{k_{n}} \int_{I_{n, r}}\left(K_{n}(x, t)-K_{n}\left(x, x_{n, r}\right)\right) \mathrm{d} t\right| & \leq k_{n}^{-2} \int_{0}^{1}\left|K_{n}^{\prime \prime}(x, t)\right| \mathrm{d} t . \\
& =O\left(k_{n}^{-2} l_{n}^{2} \log \left(l_{n}\right)\right),
\end{aligned}
$$

which, combined with (iv') gives the result. 


\section{ApPendix: Limit TheOREMS FOR CONDITIONALLY INDEPENDENT RANDOM SUM}

This part is devoted to provide general results about the central limit property and the moderate deviations principle of a sequence of random $\mathbb{R}^{p}$ valued vectors which have been used in our proofs (see also [14] and [28]). Consider a triangular array $\left(\zeta_{n, r}\right)_{r \leq k_{n}}$ of real valued random variables defined on a common probability space $(\Omega, \mathcal{A}, \mathbb{P})$ and set, for $n \geq 1$,

$$
\theta_{n}=\sum_{r=1}^{k_{n}} W_{n, r} \zeta_{n, r}
$$

where $\left(W_{n, r}\right)_{r \leq k_{n}} \subset \mathbb{R}^{p}$. In the following, for all $u \in \mathbb{R}^{p}$, we set

$$
w_{n, r}(u)=\left\langle W_{n, r}, u\right\rangle_{\mathbb{R}^{d}}^{2} \text { and } \theta_{n}(u)=\sum_{r=1}^{k_{n}} W_{n, r}(u) \zeta_{n, r}
$$

In order to state central limit theorem and moderate deviations principle for $\left(\theta_{n}\right)$ we consider a sequence $\left(\varepsilon_{n}\right)_{n \geq 1} \subset \mathbb{R}^{+}$such that $\left(\varepsilon_{n}\right)_{n \geq 1} \equiv 1$ or $\varepsilon_{n} \downarrow 0$ and we assume:

(A.1) $k_{n} \underset{n \rightarrow \infty}{\rightarrow} 0$ and $\varepsilon_{n}^{-1 / 2} w_{n}(u) \underset{n \rightarrow \infty}{\rightarrow} 0$ where $w_{n}(u):=\max _{r \leq k_{n}}\left|w_{n, r}(u)\right|$.

(A.2) There exists a covariance matrix $\Sigma$ in $\mathbb{R}^{p}$ such that for all $u \in \mathbb{R}^{p}$,

$$
\sum_{r=1}^{k_{n}} w_{n, r}^{2}(u) \underset{n \rightarrow \infty}{\rightarrow}{ }^{t} u \Sigma u:=\sigma^{2}(u)
$$

(A.3) For all $n \geq 1,\left(\zeta_{n, r}\right)_{r \leq k_{n}}$ are independent conditionally to a sigma field $\mathcal{B}_{n} \subset \mathcal{A}$.

For all $n \geq 1$, there exists a set $B_{n} \in \mathcal{B}_{n}$ such that:

(A.4) $\limsup _{n \rightarrow \infty} \varepsilon_{n} \log \mathbb{P}\left(B_{n}^{c}\right)=-\infty$.

(A.5) For all $r \leq k_{n}, \mathbb{E}\left(\zeta_{n, r} \mid \mathcal{B}_{n}\right) \mathbf{1}_{B_{n}}=0$ a.s.

(A.6) For some sequence $\eta_{n} \downarrow 0, \max _{1 \leq r \leq k_{n}}\left|\mathbb{E}\left(\zeta_{n, r}^{2} \mid \mathcal{B}_{n}\right)-1\right| \mathbf{1}_{B_{n}} \leq \eta_{n}$ a.s.

(A.7) For some real $K_{7}>0, \limsup _{n \rightarrow \infty} \max _{1 \leq r \leq k_{n}} \mathbb{E}\left(\left|\zeta_{n, r}\right|^{3} \mid \mathcal{B}_{n}\right) \mathbf{1}_{B_{n}}<K_{7}$ a.s.

(A.8) For some real $K_{8}>0$, $\limsup _{n \rightarrow \infty} \max _{l \geq 3} \max _{1 \leq r \leq k_{n}}\left(\frac{1}{l !}\right)^{1 / l} \mathbb{E}\left(\left|\zeta_{n, r}\right|^{l} \mid \mathcal{B}_{n}\right)^{1 / l} \mathbf{1}_{B_{n}}<K_{8}$ a.s.

We are now in position to express the main results of this section:

Theorem 5.1. If assumptions (A.1)-(A.7) hold with $\varepsilon_{n}=1$, then $\theta_{n} \underset{\mathcal{D}}{\rightarrow} N(0, \Sigma)$.

Proof. For all $u$ in $\mathbb{R}^{p}$, set

$$
\sigma_{n}^{2}(u):=\left(\sum_{r=1}^{k_{n}} w_{n, r}^{2}(u) \mathbb{E}\left(\zeta_{n, r}^{2} \mid \mathcal{B}_{n}\right)\right) \text { and } \tau_{n}(u):=\sum_{r=1}^{k_{n}}\left|w_{n, r}(u)\right|^{3} \mathbb{E}\left(\left|\zeta_{n, r}\right|^{3} \mid \mathcal{B}_{n}\right)
$$

By the Berry Esseen Theorem (see [30] Th. 5.4) we get for an universal constant $C_{1}$ :

$$
\sup _{x \in \mathbb{R}}\left|\mathbb{P}\left(\sigma_{n}^{-1}(u) \theta_{n}(u) \leq x \mid \mathcal{B}_{n}\right)-\Phi(x)\right| \mathbf{1}_{B_{n}} \leq C_{1} \mathbb{E}\left(\sigma_{n}^{-3}(u) \tau_{n}(u) \mid \mathcal{B}_{n}\right) \mathbf{1}_{B_{n}}
$$


where $\Phi$ denotes the standard normal distribution function. Now, by (A.2) and (A.6),

$$
\left|\sigma_{n}^{2}(u)-\sigma(u)^{2}\right| \mathbf{1}_{B_{n}} \leq\left|\sum_{r=1}^{k_{n}} w_{n, r}^{2}(u)-\sigma^{2}(u)\right|+\eta_{n} \sum_{r=1}^{k_{n}} w_{n, r}^{2}(u)=o(1) .
$$

In particular, for some real sequence $\gamma_{n} \downarrow 0$,

$$
\sigma_{n}(u) \mathbf{1}_{B_{n}} \in\left[\left(1-\gamma_{n}\right) \sigma(u) \mathbf{1}_{B_{n}},\left(1+\gamma_{n}\right) \sigma(u) \mathbf{1}_{B_{n}}\right] .
$$

Moreover, by (A.7),

$$
\tau_{n}(u) \mathbf{1}_{B_{n}} \leq w_{n}(u) K_{7} \sum_{r=1}^{k_{n}} w_{n, r}^{2}(u) .
$$

Hence, for some $C_{2}$ all $n \geq 1$ and all $x \in \mathbb{R}$,

$$
\left|\mathbb{P}\left(\sigma_{n}^{-1}(u) \theta_{n}(u) \leq x \mid \mathcal{B}_{n}\right)-\Phi(x)\right| \mathbf{1}_{B_{n}} \leq C_{2} w_{n}(u)
$$

and $\sigma_{n}^{-1}(u) \theta_{n}(u) \underset{\mathcal{D}}{\rightarrow} N(0,1)$, since

$$
\begin{aligned}
\left|\mathbb{P}\left(\sigma_{n}^{-1}(u) \theta_{n}(u) \leq x\right)-\Phi(x)\right| & \leq\left|\mathbb{P}\left(\sigma_{n}^{-1}(u) \theta_{n}(u) \leq x ; B_{n}\right)-\Phi(x) \mathbb{P}\left(B_{n}\right)\right|+\mathbb{P}\left(B_{n}^{c}\right) \\
& \leq \mathbb{E}\left(\left|\mathbb{P}\left(\sigma_{n}^{-1}(u) \theta_{n}(u) \leq x \mid \mathcal{B}_{n}\right)-\Phi(x)\right| \mathbf{1}_{B_{n}}\right)+\mathbb{P}\left(B_{n}^{c}\right) \\
& \leq C_{2} w_{n}(u)+\mathbb{P}\left(B_{n}^{c}\right)=o(1) \text { by (A.2) and (A.4). }
\end{aligned}
$$

Moreover, by (58), $\sigma_{n}(u) \stackrel{\mathbb{P}}{\rightarrow} \sigma(u)$ and we get the result by [3] Theorems $4-1$ and 7-7.

In order to deal with moderate deviation principle we assume that $\Sigma$ is regular and we define

$$
\mathrm{I}: s \in \mathbb{R}^{p} \mapsto 2^{-1 t} s \Sigma^{-1} s .
$$

Theorem 5.2. Assume that $\Sigma$ is regular and (A.1)-(A.8) hold for $\varepsilon_{n} \downarrow 0$. Then, $\left(\varepsilon_{n}^{1 / 2} \theta_{n}\right) \in \operatorname{LDP}\left(\varepsilon_{n}, \mathrm{I}\right)$.

Proof. Using the conditional independance of $\left(\zeta_{n, r}\right)_{r \leq k_{n}}$ and (A.5), we get that

$$
\mathbb{E}\left(\exp \left(\varepsilon_{n}^{-1 / 2} \theta_{n}(u) \mathbf{1}_{B_{n}}\right) \mid \mathcal{B}_{n}\right)=\mathbf{1}_{B_{n}^{c}}+\prod_{r \leq k_{n}}\left(1+2^{-1} \varepsilon_{n}^{-1} w_{n, r}^{2}(u)\left(1+\Gamma_{n, r}(u)\right)\right) \mathbf{1}_{B_{n}},
$$

where

$$
\Gamma_{n, r}(u):=\left(\mathbb{E}\left(\zeta_{n, r}^{2} \mid \mathcal{B}_{n}\right)-1+2 \sum_{l \geq 3}(l !)^{-1}\left(\varepsilon_{n}^{-1 / 2} w_{n, r}(u)\right)^{l-2} \mathbb{E}\left(\zeta_{n, r}^{l} \mid \mathcal{B}_{n}\right)\right) \mathbf{1}_{B_{n}} .
$$

Now, for all large $n,($ A.6) and (A.8) lead to

$$
\begin{aligned}
\max _{r \leq k_{n}}\left|\Gamma_{n, r}(u)\right| & \leq \eta_{n}+2 K_{8}^{2} \sum_{l \geq 3}\left|K_{8} \varepsilon_{n}^{-1 / 2} w_{n, r}(u)\right|^{l-2} \\
& \leq \eta_{n}+4 K_{8}^{3} \varepsilon_{n}^{-1 / 2} w_{n}(u):=\gamma_{n}(u)=o(1) .
\end{aligned}
$$


Hence,

$$
\begin{aligned}
\mathbb{E}\left(\exp \left(\varepsilon_{n}^{-1 / 2} \theta_{n}(u) \mathbf{1}_{B_{n}}\right)\right) & \geq \mathbb{E}\left(\mathbf{1}_{B_{n}^{c}}+\left(\prod_{r \leq k_{n}}\left(1+2^{-1} \varepsilon_{n}^{-1} w_{n, r}^{2}(u)\left(1-\gamma_{n}(u)\right)\right)\right) \mathbf{1}_{B_{n}}\right) \\
& \geq \mathbb{P}\left(B_{n}\right) \prod_{r \leq k_{n}}\left(1+2^{-1} \varepsilon_{n}^{-1} w_{n, r}^{2}(u)\left(1-\gamma_{n}(u)\right)\right) \\
\mathbb{E}\left(\exp \left(\varepsilon_{n}^{-1 / 2} \theta_{n}(u) \mathbf{1}_{B_{n}}\right)\right) & \leq \mathbb{E}\left(\mathbf{1}_{B_{n}^{c}}+\left(\prod_{r \leq k_{n}}\left(1+2^{-1} \varepsilon_{n}^{-1} w_{n, r}^{2}(u)\left(1+\gamma_{n}(u)\right)\right)\right) \mathbf{1}_{B_{n}}\right) \\
& \leq \mathbb{P}\left(B_{n}^{c}\right)+\prod_{r \leq k_{n}}\left(1+2^{-1} \varepsilon_{n}^{-1} w_{n, r}^{2}(u)\left(1+\gamma_{n}(u)\right)\right),
\end{aligned}
$$

and, therefore,

with

$$
l_{n, 1}(u) \leq \varepsilon_{n} \log \mathbb{E}\left(\exp \left(\varepsilon_{n}^{-1 / 2} \theta_{n}(u) \mathbf{1}_{B_{n}}\right)\right) \leq l_{n, 2}(u)
$$

and

$$
l_{n, 1}(u)=\varepsilon_{n} \log \mathbb{P}\left(B_{n}\right)+\sum_{r \leq k_{n}} \varepsilon_{n} \log \left(1+2^{-1} \varepsilon_{n}^{-1} w_{n, r}^{2}(u)\left(1-\gamma_{n}(u)\right)\right)
$$

$$
l_{n, 2}(u)=\varepsilon_{n} \log \left(\mathbb{P}\left(B_{n}^{c}\right)+\prod_{r \leq k_{n}}\left(1+2^{-1} \varepsilon_{n}^{-1} w_{n, r}^{2}(u)\left(1+\gamma_{n}(u)\right)\right)\right) .
$$

Now, using (59) and Taylor expension we get,

$$
\lim _{n \rightarrow \infty} \varepsilon_{n} \log \mathbb{E}\left(\exp \left(\varepsilon_{n}^{-1 / 2} \theta_{n}(u) \mathbf{1}_{B_{n}}\right)\right)=2^{-1 t} u \Sigma u,
$$

hence, by the Gartner-Ellis Theorem (see [7] Th. 2-3-6), $\left(\varepsilon_{n}^{1 / 2} \theta_{n} \mathbf{1}_{B_{n}}\right)_{n \geq 1} \in \operatorname{LDP}\left(\varepsilon_{n}, \mathrm{I}\right)$.

Finally, since, for all $\eta>0$,

$$
\limsup _{n \rightarrow \infty} \varepsilon_{n} \log \mathbb{P}\left(\varepsilon_{n}^{1 / 2}\left\|\theta_{n}-\theta_{n} \mathbf{1}_{B_{n}}\right\|_{\mathbb{R}^{p}} \geq \eta\right) \leq \limsup _{n \rightarrow \infty} \varepsilon_{n} \log \mathbb{P}\left(B_{n}^{c}\right)=-\infty
$$

we get the intended result by [7] Theorem 4-2-21.

\section{REFERENCES}

[1] J.A. Adell and P. Jodrá, The median of the Poisson distribution. Metrika 613 (2005) 337-346.

[2] P. Baufays and J.-P. Rasson, A new geometric discriminant rule. Comput. Stat. Q. 2 (1985) 15-30.

[3] P. Billingsley, Convergence of Probability measures. Wiley (1968).

[4] D. Deprins, L. Simar and H. Tulkens, Measuring Labor Efficiency in Post Offices, in The Performance of Public Enterprises: Concepts and Measurements, M. Marchand, P. Pestieau and H. Tulkens Eds., North Holland, Amsterdam (1984).

[5] J.D. Deuschel and D.W. Stroock, Large Deviations. Pure and Applied Mathematics, 137. Boston, MA Academic Press (1989).

[6] L.P. Devroye and G.L. Wise, Detection of abnormal behavior via non parametric estimation of the support. SIAM J. Appl. Math. 38 (1980) 448-480.

[7] A. Dembo and O. Zeitouni, Large Deviations Techniques and Applications. Jones and Bartlett, Boston and London (1993).

[8] L. Gardes, Estimating the support of a Poisson process via the Faber-Schauder basis and extrems values. Publications de l'Institut de Statistique de l'Université de Paris XLVI 43-72 (2002).

[9] J. Geffroy, Sur un problème d'estimation géométrique. Publications de l'Institut de Statistique de l'Université de Paris XIII (1964) 191-200. 
[10] I. Gijbels, E. Mammen, B.U. Park and L. Simar, On estimation of monotone and concave frontier functions. J. Amer. Statist. Assoc. 94 (1999) 220-228.

[11] S. Girard and P. Jacob, Projection estimates of point processes boundaries. J. Statist. Planning Inference 116 (2003), 1-15.

[12] S. Girard and P. Jacob, Extreme values and kernel estimates of point processes boundaries. ESAIM: PS 8 (2005) 150-168.

[13] S. Girard and L. Menneteau, Central limit theorems for smoothed extreme value estimates of Poisson point processes boundaries. J. Statist. Planning Inference 135 (2005) 433-460.

[14] S. Girard and L. Menneteau, Smoothed extreme value estimators of non uniform boundaries with applications to star-shaped supports estimation. Submitted.

[15] A. Hardy and J.P. Rasson, Une nouvelle approche des problèmes de classification automatique. Statist. Anal. Données 7 (1982) $41-56$.

[16] P. Hall, M. Nussbaum and S.E. Stern, On the estimation of a support curve of indeterminate sharpness. J. Multivariate Anal. 62 (1997) 204-232.

[17] P. Hall, B.U. Park and S.E. Stern, On polynomial estimators of frontiers and boundaries. J. Multivariate Anal. 66 (1998) $71-98$.

[18] W. Härdle, Applied nonparametric regression. Cambridge University Press, Cambridge (1990).

[19] W. Härdle, P. Hall and L. Simar, Iterated bootstrap with application to frontier models. J. Productivity Anal. 6 (1995) 63-76.

[20] W. Härdle, B.U. Park and A.B. Tsybakov, Estimation of a non sharp support boundaries. J. Multivariate Anal. 43 (1995) $205-218$.

[21] J.A. Hartigan, Clustering Algorithms. Wiley, Chichester (1975).

[22] W. Kallenberg, Intermediate efficiency theory and examples. Ann. Statist. 11 (1983) 170-182.

[23] W. Kallenberg, On moderate deviation theory in estimation. Ann. Statist. 11 (1983) 498-504.

[24] A.P. Korostelev, L. Simar and A.B. Tsybakov, Efficient estimation of monotone boundaries. Ann. Statist. 23 (1995) $476-489$.

[25] A.P. Korostelev and A.B. Tsybakov, Minimax theory of image reconstruction, in Lecture Notes in Statistics 82, SpringerVerlag, New York (1993).

[26] A.P. Korostelev and A.B. Tsybakov, Asymptotic efficiency of the estimation of a convex set. Problems Inform. Transmission 30 (1994) 317-327.

[27] E. Mammen and A.B. Tsybakov, Asymptotical minimax recovery of sets with smooth boundaries. Ann. Statist. 23 (1995) $502-524$.

[28] L. Menneteau, Limit theorems for piecewise constant kernel smoothed estimates of point process boundaries. Technical Report (2007).

[29] A. Mokkadem and M. Pelletier, Moderate deviations for the kernel mode estimator and some applications. J. Statist. Planning Inference 135 (2005) 276-299.

[30] V.V. Petrov, Limit theorems of probability theory. Sequences of independent random variables. Oxford Studies in Probability, (1995) 4.

[31] G.R. Shorack and J.A. Wellner, Empirical processes with applications to statistics. Wiley, New York (1986).

[32] G.P. Tolstov, Fourier series. 2nd ed. New York: Dover Publications (1976).

[33] A.B. Tsybakov, On nonparametric estimation of density level sets. Ann. Statist. 25 (1997) 948-969. 